\title{
Wear Processes in a Mechanical Friction Clutch: Theoretical, Numerical, and Experimental Studies
}

\author{
Dariusz Grzelczyk and Jan Awrejcewicz \\ Department of Automation, Biomechanics and Mechatronics, Lodz University of Technology, 1/15 Stefanowski Street, \\ 90-924 Lodz, Poland \\ Correspondence should be addressed to Dariusz Grzelczyk; dariusz.grzelczyk@p.lodz.pl
}

Received 8 January 2015; Accepted 6 March 2015

Academic Editor: N. B. Naduvinamani

Copyright (C) 2015 D. Grzelczyk and J. Awrejcewicz. This is an open access article distributed under the Creative Commons Attribution License, which permits unrestricted use, distribution, and reproduction in any medium, provided the original work is properly cited.

\begin{abstract}
Mathematical modeling, theoretical/numerical analysis, and experimental verification of wear processes occurring on the contact surface of friction linings of a mechanical friction clutch are studied. In contrast to many earlier papers we take into consideration wear properties and flexibility of friction materials being in friction contact. During mathematical modeling and numerical simulations we consider a general nonlinear differential model of wear (differential wear model) and a model of wear in the integral form (integral wear model). Equations governing contact pressure and wear distributions of individual friction linings, decrease of distance between clutch shields, and friction torque transmitted by the clutch are derived and compared with experimental data. Both analytical and numerical analyses are carried out with the qualitative and quantitative theories of differential and integral equations, including the Laplace transform approach to ODEs. We show that theoretical results and numerical simulations agree with the experimental data. Finally, a numerical analysis of the proposed mathematical models was carried out in a wider range of parameters of the considered system.
\end{abstract}

\section{Introduction}

A clutch is an important element used for coupling shafts in many mechanical systems and transmitting torque between them. In the early period of development of transport, mechanical industry, and machine engineering, both belts and transmissions were used to transmit torque between shafts functioning together. However, a need for individual power transmissions and a compact coupling of shafts influenced more advanced modeling of the processes in mechanical friction clutches. The first and the oldest simple clutches were used for direct connection of coaxial shafts; however the present-day demands and future trends in the technology of clutch production pose numerous demands with regard to their structure, strength, functioning, and life. For this reason, basic issues in designing power transmission systems include an increase of productivity and improvement of functioning quality of driven machines, enhancement of the degree of reliability, and obtaining better technoeconomic indices of these systems. To satisfy these assumptions in mechanical friction clutches, we should have appropriate knowledge on mathematical modeling and description of this system including contact phenomena (friction, wear, and heat production) occurring therein. The so far mentioned complex approach allows for a better prediction of the real systems behavior. The so far discussed phenomena occur not only in various mechanical friction connections (bars, bearings, gears, guides, transmissions, clutches, brakes, and others), but also in every motion in nature and in everyday life. These phenomena take place at the contact surfaces of bodies being in friction contact and rubbing against each other. The science dedicated to these phenomena which occur during friction contact of solid bodies is known as tribology. Tribology has a long history, and its origins and development were described in detail by Dowson in the extensive work History of Tribology [1].

\section{Motivation and Goals of the Work}

Issues related to the interaction of dynamics, contact phenomena, and accompanying tribological processes in various 
types of mechanical systems have been the object of interest and investigations of many researchers for many years. The aforementioned issues have been considered in various friction connections, like bars, transmissions, gears, guides, bearings, clutches, brakes, and others. In the investigations devoted to dynamics, contact phenomena and tribological processes occurring in the systems with a friction clutch implied derivation of numerous and different mathematical models. Often, wear processes were not taken into account while studying the system dynamics. Simultaneously, a typical analysis of the mentioned phenomena and processes did not include inertia of contacting bodies. Moreover, in most cases simplified mathematical models were used therein and in addition (as a rule) separately for particular issues and without mutual interactions among them.

In this paper, an attempt of strict mathematical description of the mentioned individual issues has been presented. Namely, (i) the considered friction clutch has been treated as a friction connection of elastic bodies taking into account flexibility of the material of friction linings in axial direction; (ii) a general nonlinear differential wear model, where wear is modeled via the power-type function of contact pressure and relative sliding velocity with rates dependent on the model of wear, the step of lubricating, and spreading on contacting surfaces, and an integral wear model taking into consideration hereditary and memory processes (the gradual decrease of speed of wear of friction linings as a result of abrasive adapting to each other in the process of the exploitation) have been used in numerical simulations; (iii) nonuniform contact pressure distributions on the contact surface of clutch friction linings have been determined for any instant of time; (iv) for any instant of time nonuniform distributions of wear of frictional linings of contacting bodies have been calculated; (v) changes of friction torque transmitted by the clutch resulting from the change of contact pressure distribution have been computed. The above presented attempt at joining the mentioned issues into one complex tribological system is not an easy task; however the results obtained in this way should enable better projection of the behavior of this type of actual systems.

\section{Literature Review}

Wear is a dynamical process [2] related to changes in the surfaces of bodies moving relative to one another as a result of mechanical interaction between them. This process depends on many factors and parameters (geometry of contacting surfaces, normal applied force, relative velocity, material hardness, etc. [3]). Wear of mechanical parts in most cases is considered to be the main cause of the deterioration of the quality of operation and performance of a device. Studies on the wear process and its modeling have been carried out for many years [4]. One of the first scientists investigating wear processes was Archard, who proposed a linear model of wear for metals [5]. In his model wear was measured by volume divided by the distance of acting friction force and was defined as a wear coefficient, which depended on both loading force and hardness of the material.
This model was used for metals by the authors of works [6-9] and others. In turn, one of the first researchers who proposed a nonlinear wear model for friction material of brakes was Rhee $[10,11]$. His groundbreaking work inspired other researchers to conduct further studies of these systems $[12,13]$. Since then, many mathematical models describing wear processes in friction joints of different type, in different external conditions and for various materials, have come into being yet. In the literature related to tribology about three hundred various models of wear may be found, from simple empirical equations to complex mathematical relations [3]. However, a general mathematical model which adequately describes any issue related to the wear processes has not been proposed so far. Various proposed simple and more complex wear models describe different wear processes with sufficient accuracy only for a selected pair of friction materials, the geometry of the system, or the external process conditions [14]. Numerical calculations of wear processes in various types of friction connections can be found in numerous works, for instance $[3,6-9,15-22]$ and many others.

Friction phenomena occurring at working surfaces of the clutch accompany wear processes of materials of frictional linings. A dominant character of wear of clutch linings is an abrasive tribological wear process, while other types of wear processes can be neglected. In general, it is assumed that the wear of friction working surfaces of the clutch is proportional to the work of friction force. In a simplified way, strength $L$ of the clutch friction surfaces can be estimated via the relation

$$
L=\frac{V_{v}}{q_{v} L_{t} m_{w}},
$$

where $V_{v}$ is the volume wear, $q_{v}$ is the specific wear parameter characterizing the material of lining, and $L_{t}$ is the work of friction force during a single association of the clutch, while $m_{w}$ is the number of starts of the clutch per hour. Maximum volume wear $V_{v}$ may be determined if it is assumed to be acceptable to reduce the thickness of friction linings $s_{s}$, usually from 0.8 to 0.9 thickness of the lining; thus

$$
V_{v}=A_{s} s_{s}
$$

where $A_{s}$ is the working friction surface of the clutch.

There exist a few wear models depending on the applied friction description. According to Archard [5], the model of wear written in the differential form takes the following form:

$$
\frac{d w(t)}{d t}=K^{(w)}\left|V_{r}(t)\right| P(t),
$$

where $t$ denotes time, $w(t)$ is the wear, $K^{(w)}$ is the material wear coefficient, and $V_{r}(t)$ is the relative velocity of rubbing surfaces, while $P(t)$ is the contact pressure between them. It is the linear model of wear which considers contact pressure and relative velocity of rubbing surfaces. This model of wear was used in [15] to calculate wear in the mechanical thermoelastic contact of a solid isotropic circular shaft (cylinder) with a cylindrical tube-like rigid bush (rigid ring). In other types of friction connections this model was also used in $[18,19,23,24]$. 
In this paper, in modeling wear processes of clutch friction linings the general nonlinear differential model of wear has been used, governed by [21]

$$
\frac{d w(t)}{d t}=K^{(w)}\left(T^{\prime}(t)\right)\left|V_{r}(t)\right|^{\beta} P^{\alpha}(t)
$$

where wear coefficient $K^{(w)}\left(T^{\prime}(t)\right)$ is the function of temperature $T^{\prime}(t)$ on the contact surface and $\alpha$ and $\beta$ coefficients are the quantities dependent on the model of wear, grade of machining, and lubrication of rubbing surfaces. Thus, it is the nonlinear model of wear, wherein the speed of wear is the nonlinear (power) function of contact pressure and relative velocity of rubbing surfaces. The presented nonlinear model of wear (4) was used earlier in works [21, 25] and others.

For the above presented wear models in stationary conditions (constant normal force, constant relative sliding velocity, constant contact pressure, and wear coefficient independent of temperature) the speed of wear is constant $(d w(t) / d t=$ constant $)$. At variable conditions of external load the so-called delay effects may be observed [21, 22, 26]. For some frictional materials, in spite of stable conditions of wear processes, the wear coefficient changes with time as a result of ageing or wearing of friction linings. Then, there is a necessity to use other wear models than these presented above. An adequate mathematical description of such a wear process is the integral model of wear in the form of $[17,21]$

$$
w(t)=\int_{0}^{t} K^{(w)}\left(T^{\prime}\left(t^{\prime}\right)\right)\left|V_{r}\left(t^{\prime}\right)\right| K^{\prime}\left(t, t^{\prime}\right) P\left(t^{\prime}\right) d t^{\prime}
$$

where $K^{\prime}\left(t, t^{\prime}\right)=K_{1}^{\prime}\left(t^{\prime}\right) K_{2}^{\prime}\left(t-t^{\prime}\right)$, wherein $K_{1}^{\prime}\left(t^{\prime}\right)=1+$ $c \exp \left(-\gamma^{\prime} t^{\prime}\right)$ and $K_{2}^{\prime}\left(t-t^{\prime}\right)=1-\exp \left[-\gamma^{\prime \prime}\left(t-t^{\prime}\right)\right]$ are the so-called hereditary and memory kernels. Exponential functions in the presented wear model with parameters $c, \gamma^{\prime}$, and $\gamma^{\prime \prime}$ are responsible for decreasing the speed of wear process also in stationary conditions. Model of wear (5) for $K_{1}^{\prime}\left(t^{\prime}\right)=1$ and $K_{2}^{\prime}\left(t-t^{\prime}\right)=1-\exp \left[-\gamma^{\prime \prime}\left(t-t^{\prime}\right)\right]$ was used in $[22,27]$. For a model of contact of a thermoelastic layer with a thermally insulated plate the solution was obtained taking into account both wear and heat processes. In [28], integral wear model (5) was used in the system of elliptical friction contact taking into consideration wear process for constant wear coefficient, constant relative sliding velocity, and constant contact pressure. In [16] this model was used to determine the wear of an elastic and heat transferring cylinder inserted into the bush (rigid ring) for a constant wear coefficient. In [20] the integral wear model was used in the system of two contacting elastic and heat conducting layers, wherein nonconstant (depending on the temperature of contact surfaces calculated in the same mathematical model) wear coefficient was taken.

As mentioned in Section 2, the considered problems should enable better projection of the behavior of type of the considered actual systems. From practical point of view, to minimize the maximal contact pressure between contacting bodies usually the appropriate contact shape is determined. Moreover, minimization of wear volume rate, minimization of friction dissipation power, or minimization of the wear dissipation power belongs to other classes of optimization problems. In [29] the variational formulation of contact shape evolution associated with the wear process was discussed. Friction contact of two bodies was considered and analysed in the case of constant relative sliding velocity between them, as well as in the case when one of the bodies rotates with respect to another body. In the mentioned paper it is demonstrated that the wear dissipation power at the contact surface is minimal in the steady state of wear process. In [30], as an example of steady wear state, the analysis of disk and drum brakes is presented, whereas in [31] of the same authors wear analysis of a punch translating on an elastic strip and wear induced by a rotating punch on a toroidal surface is considered as an example of the analysis of coupled thermoelastic steady wear regimes. The two mentioned above papers present that the temperature field generated by frictional and wear dissipation on the contact surface is assumed to reach a steady state. The steady state is assumed to correspond to minimum of the wear dissipation power. Some information on the contact optimization problems was presented in [29]. An extensive literature review of contact pressure optimization problems can be found in [32].

\section{Mathematical Models}

In this section the considered mechanical friction clutch and mathematical models (both differential and integral wear model) describing wear processes are presented.

4.1. Model of the Considered Mechanical Friction Clutch. We are focused on the mechanical friction clutch shown in Figure 1. This figure presents a model of mechanical friction clutch and a cross section of friction linings of this clutch with a computational grid (plotted on the cross section of the linings divided into $m$ equal segments (sections) along radius $R$ ), in nodes whose appropriate pressures and wears of individual linings are being calculated. The friction linings are fixed to both shields of the clutch. The friction contact between linings occurs in the ring area $R \in\left[R_{1}, R_{2}\right]$. The mentioned shields are being pressed by axial force $Q(t)$ and their relative angular velocity is equal to $\Omega_{r}(t)$, while contact pressure at any contact point and time is equal to $P(R, t)$. Material wear coefficients for the upper and lower lining, dependent on temperature $T^{\prime}(R, t)$ in a given contact point and time, are denoted by $K_{1}^{(w)}\left(T^{\prime}(R, t)\right)$ and $K_{2}^{(w)}\left(T^{\prime}(R, t)\right)$, respectively. In turn, stiffness coefficients of friction materials of these linings in axial direction are $k_{1}$ and $k_{2}$, respectively.

4.2. Mathematical Modeling of Wear Processes. We assume the elastic contact interaction between pressed friction linings to satisfy Winkler relations. According to the mentioned relations, at each point of the contact surface the elastic boundary displacements $U^{(1)}(R, t)$ for the upper friction linings and $U^{(2)}(R, t)$ for the lower one, along the axis perpendicular to the contact surface, have the form $[33,34]$

$$
U^{(1)}(R, t)=k_{1} P(R, t), \quad U^{(2)}(R, t)=k_{2} P(R, t) .
$$




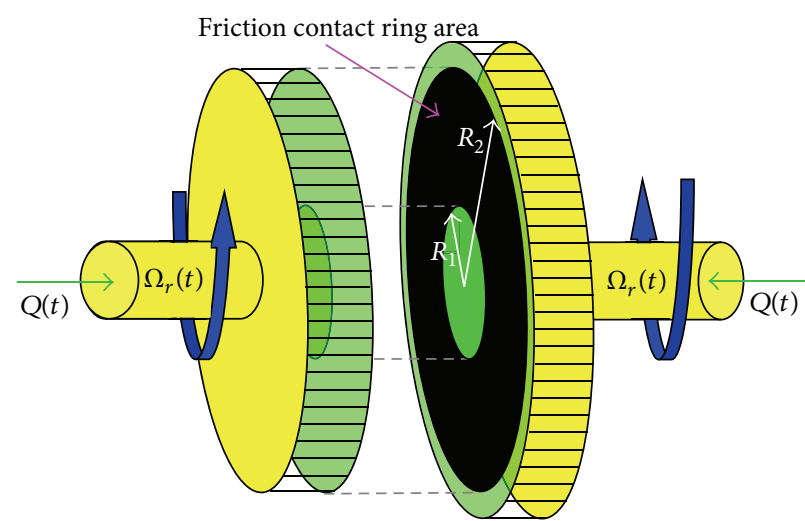

(a)

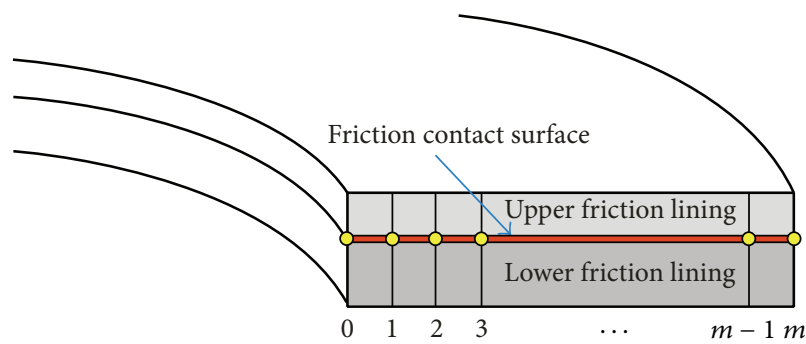

(b)

FIGURE 1: Model of the considered mechanical friction clutch (a) and the cross section of friction linings with a plotted computational grid (b).

To obtain analytical results similar approach was also used, for instance, in [31], where the numerical analysis of steady wear regimes induced by relative sliding of two contacting bodies has been presented.

The equations describing wears $U_{1}^{(w)}(R, t)$ of the upper friction lining and $U_{2}^{(w)}(R, t)$ of the lower one at each point of contact for any time instant and for $V_{r}(R, t)=\Omega_{r}(t) R$ in the case of differential wear model $(4)$ have the form $[21,25]$

$$
\begin{aligned}
& \frac{\partial U_{1}^{(w)}(R, t)}{\partial t}=K_{1}^{(w)}\left(T^{\prime}(R, t)\right)\left|\Omega_{r}(t)\right|^{\beta_{1}} R^{\beta_{1}} P^{\alpha_{1}}(R, t), \\
& \frac{\partial U_{2}^{(w)}(R, t)}{\partial t}=K_{2}^{(w)}\left(T^{\prime}(R, t)\right)\left|\Omega_{r}(t)\right|^{\beta_{2}} R^{\beta_{2}} P^{\alpha_{2}}(R, t) ;
\end{aligned}
$$

and in the case of integral wear model (5) have the following form [17, 21]:

$$
\begin{aligned}
& U_{1}^{(w)}(R, t)= \int_{0}^{t} K_{1}^{(w)}\left(T^{\prime}\left(R, t^{\prime}\right)\right)\left|\Omega_{r}\left(t^{\prime}\right)\right| R K_{1}^{\prime}\left(t, t^{\prime}\right) \\
& \cdot P\left(R, t^{\prime}\right) d t^{\prime}, \\
& U_{2}^{(w)}(R, t)=\int_{0}^{t} K_{2}^{(w)}\left(T^{\prime}\left(R, t^{\prime}\right)\right)\left|\Omega_{r}\left(t^{\prime}\right)\right| R K_{2}^{\prime}\left(t, t^{\prime}\right) \\
& \cdot P\left(R, t^{\prime}\right) d t^{\prime} .
\end{aligned}
$$

Conditions of the contact of linings in the clutch (at each point of the surface) are as follows [33, 34]:

$$
\begin{gathered}
U^{(1)}(R, t)+U^{(2)}(R, t)+U_{1}^{(w)}(R, t)+U_{2}^{(w)}(R, t)=E(t), \\
\Longrightarrow \frac{\partial U^{(1)}(R, t)}{\partial t}+\frac{\partial U^{(2)}(R, t)}{\partial t}+\frac{\partial U_{1}^{(w)}(R, t)}{\partial t} \\
\quad+\frac{\partial U_{2}^{(w)}(R, t)}{\partial t}=\frac{d E(t)}{d t},
\end{gathered}
$$

where $E(t)$ is the function describing the decrease of distance between clutch shields. Taking into account relations (6)-(8) in (9) and $k_{1}+k_{2}=k$, we obtain

$$
\begin{aligned}
& k \frac{\partial P(R, t)}{\partial t}+K_{1}^{(w)}\left(T^{\prime}(R, t)\right)\left|\Omega_{r}(t)\right|^{\beta_{1}} R^{\beta_{1}} P^{\alpha_{1}}(R, t) \\
& \quad+K_{2}^{(w)}\left(T^{\prime}(R, t)\right)\left|\Omega_{r}(t)\right|^{\beta_{2}} R^{\beta_{2}} P^{\alpha_{2}}(R, t)=\frac{d E(t)}{d t}
\end{aligned}
$$

for the differential wear model and

$$
\begin{aligned}
& k P(R, t)+\int_{0}^{t} K_{1}^{(w)}\left(T^{\prime}\left(R, t^{\prime}\right)\right)\left|\Omega_{r}\left(t^{\prime}\right)\right| R K_{1}^{\prime}\left(t, t^{\prime}\right) \\
& \quad \cdot P\left(R, t^{\prime}\right) d t^{\prime} \\
& \quad+\int_{0}^{t} K_{2}^{(w)}\left(T^{\prime}\left(R, t^{\prime}\right)\right)\left|\Omega_{r}\left(t^{\prime}\right)\right| R K_{2}^{\prime}\left(t, t^{\prime}\right) P\left(R, t^{\prime}\right) d t^{\prime} \\
& =E(t)
\end{aligned}
$$

for the integral wear model. Multiplying (10) and (11) by $R d R$ and next integrating in the area $R \in\left[R_{1}, R_{2}\right]$, we get the following relationship for the differential wear model:

$$
\begin{aligned}
& \int_{R_{1}}^{R_{2}} k \frac{\partial P(R, t)}{\partial t} R d R+\int_{R_{1}}^{R_{2}} K_{1}^{(w)}\left(T^{\prime}(R, t)\right)\left|\Omega_{r}(t)\right|^{\beta_{1}} R^{\beta_{1}} \\
& \cdot P^{\alpha_{1}}(R, t) R d R \\
& \quad+\int_{R_{1}}^{R_{2}} K_{2}^{(w)}\left(T^{\prime}(R, t)\right)\left|\Omega_{r}(t)\right|^{\beta_{2}} R^{\beta_{2}} P^{\alpha_{2}}(R, t) R d R \\
&=\int_{R_{1}}^{R_{2}} \frac{d E(t)}{d t} R d R ;
\end{aligned}
$$


and the appropriate relationship for the integral wear model

$$
\begin{gathered}
\int_{R_{1}}^{R_{2}} k P(R, t) R d R \\
+\int_{R_{1}}^{R_{2}}\left(\int_{0}^{t} K_{1}^{(w)}\left(T^{\prime}\left(R, t^{\prime}\right)\right)\left|\Omega_{r}\left(t^{\prime}\right)\right|\right. \\
\left.\cdot R K_{1}^{\prime}\left(t, t^{\prime}\right) P\left(R, t^{\prime}\right) d t^{\prime}\right) R d R \\
+\int_{R_{1}}^{R_{2}}\left(\int_{0}^{t} K_{2}^{(w)}\left(T^{\prime}\left(R, t^{\prime}\right)\right)\left|\Omega_{r}\left(t^{\prime}\right)\right|\right. \\
\left.=R K_{2}^{\prime}\left(t, t^{\prime}\right) P\left(R, t^{\prime}\right) d t^{\prime}\right) R d R \\
=\int_{R_{1}}^{R_{2}} E(t) R d R .
\end{gathered}
$$

A condition to be satisfied in the considered system is as follows:

$$
\begin{aligned}
Q(t) & =2 \pi \int_{R_{1}}^{R_{2}} R P(R, t) d R, \\
& \Longrightarrow \frac{d Q(t)}{d t}=2 \pi \int_{R_{1}}^{R_{2}} R \frac{\partial P(R, t)}{\partial t} d R .
\end{aligned}
$$

Taking (14) in (12), after appropriate transformations we get (in the case of differential wear model)

$$
\begin{aligned}
& \frac{k}{\pi\left(R_{2}^{2}-R_{1}^{2}\right)} \frac{d Q(t)}{d t}+\frac{2}{R_{2}^{2}-R_{1}^{2}}\left|\Omega_{r}(t)\right|^{\beta_{1}} \\
& \cdot \int_{R_{1}}^{R_{2}} K_{1}^{(w)}\left(T^{\prime}(R, t)\right) R^{1+\beta_{1}} P^{\alpha_{1}}(R, t) d R \\
& +\frac{2}{R_{2}^{2}-R_{1}^{2}}\left|\Omega_{r}(t)\right|^{\beta_{2}} \\
& \quad \cdot \int_{R_{1}}^{R_{2}} K_{2}^{(w)}\left(T^{\prime}(R, t)\right) R^{1+\beta_{2}} P^{\alpha_{2}}(R, t) d R=\frac{d E(t)}{d t} .
\end{aligned}
$$

Taking (14) in (13), after appropriate transformations in the case of integral wear model we get

$$
\begin{gathered}
\frac{k Q(t)}{\pi\left(R_{2}^{2}-R_{1}^{2}\right)}+\frac{2}{R_{2}^{2}-R_{1}^{2}} \\
\cdot \int_{R_{1}}^{R_{2}}\left(\int_{0}^{t} K_{1}^{(w)}\left(T^{\prime}\left(R, t^{\prime}\right)\right)\left|\Omega_{r}\left(t^{\prime}\right)\right| K_{1}^{\prime}\left(t, t^{\prime}\right)\right. \\
\left.\cdot P\left(R, t^{\prime}\right) d t^{\prime}\right) R^{2} d R \\
+\frac{2}{R_{2}^{2}-R_{1}^{2}} \int_{R_{1}}^{R_{2}}\left(\int_{0}^{t} K_{2}^{(w)}\left(T^{\prime}\left(R, t^{\prime}\right)\right)\left|\Omega_{r}\left(t^{\prime}\right)\right|\right. \\
\left.\cdot K_{2}^{\prime}\left(t, t^{\prime}\right) P\left(R, t^{\prime}\right) d t^{\prime}\right) R^{2} d R=E(t) .
\end{gathered}
$$

Comparing relations (10) and (15) one gets

$$
\begin{aligned}
K_{1}^{(w)} & \left(T^{\prime}(R, t)\right)\left|\Omega_{r}(t)\right|^{\beta_{1}} R^{\beta_{1}} P^{\alpha_{1}}(R, t) \\
& +K_{2}^{(w)}\left(T^{\prime}(R, t)\right)\left|\Omega_{r}(t)\right|^{\beta_{2}} R^{\beta_{2}} P^{\alpha_{2}}(R, t)+k \frac{\partial P(R, t)}{\partial t} \\
= & \frac{2}{R_{2}^{2}-R_{1}^{2}}\left|\Omega_{r}(t)\right|^{\beta_{1}} \\
& \cdot \int_{R_{1}}^{R_{2}} K_{1}^{(w)}\left(T^{\prime}(R, t)\right) R^{1+\beta_{1}} P^{\alpha_{1}}(R, t) d R \\
& +\frac{2}{R_{2}^{2}-R_{1}^{2}}\left|\Omega_{r}(t)\right|^{\beta_{2}} \\
& \cdot \int_{R_{1}}^{R_{2}} K_{2}^{(w)}\left(T^{\prime}(R, t)\right) R^{1+\beta_{2}} P^{\alpha_{2}}(R, t) d R \\
& +\frac{k}{\pi\left(R_{2}^{2}-R_{1}^{2}\right)} \frac{d Q(t)}{d t} .
\end{aligned}
$$

In turn, comparison of relations (11) and (16) yields

$$
\begin{aligned}
& \int_{0}^{t} K_{1}^{(w)}\left(T^{\prime}\left(R, t^{\prime}\right)\right)\left|\Omega_{r}\left(t^{\prime}\right)\right| R K_{1}^{\prime}\left(t, t^{\prime}\right) P\left(R, t^{\prime}\right) d t^{\prime} \\
& \quad+\int_{0}^{t} K_{2}^{(w)}\left(T^{\prime}\left(R, t^{\prime}\right)\right)\left|\Omega_{r}\left(t^{\prime}\right)\right| R K_{2}^{\prime}\left(t, t^{\prime}\right) P\left(R, t^{\prime}\right) d t^{\prime} \\
& \quad+k P(R, t) \\
& =\frac{2}{R_{2}^{2}-R_{1}^{2}} \int_{R_{1}}^{R_{2}}\left(\int_{0}^{t} K_{1}^{(w)}\left(T^{\prime}\left(R, t^{\prime}\right)\right)\left|\Omega_{r}\left(t^{\prime}\right)\right| K_{1}^{\prime}\left(t, t^{\prime}\right)\right. \\
& \quad+\frac{2}{R_{2}^{2}-R_{1}^{2}} \int_{R_{1}}^{R_{2}}\left(\int_{0}^{t} K_{2}^{(w)}\left(T^{\prime}\left(R, t^{\prime}\right)\right)\left|\Omega_{r}\left(t^{\prime}\right)\right|\right. \\
& +\frac{k Q(t)}{\pi\left(R_{2}^{2}-R_{1}^{2}\right)} .
\end{aligned}
$$

Changing the order of integration in (18) gives

$$
\begin{aligned}
& \int_{0}^{t} K_{1}^{(w)}\left(T^{\prime}\left(R, t^{\prime}\right)\right)\left|\Omega_{r}\left(t^{\prime}\right)\right| R K_{1}^{\prime}\left(t, t^{\prime}\right) P\left(R, t^{\prime}\right) d t^{\prime} \\
& \quad+\int_{0}^{t} K_{2}^{(w)}\left(T^{\prime}\left(R, t^{\prime}\right)\right)\left|\Omega_{r}\left(t^{\prime}\right)\right| R K_{2}^{\prime}\left(t, t^{\prime}\right) P\left(R, t^{\prime}\right) d t^{\prime} \\
& \quad+k P(R, t) \\
& =\frac{2}{R_{2}^{2}-R_{1}^{2}} \int_{0}^{t} K_{1}^{\prime}\left(t, t^{\prime}\right)\left|\Omega_{r}\left(t^{\prime}\right)\right|
\end{aligned}
$$




$$
\begin{gathered}
\cdot\left(\int_{R_{1}}^{R_{2}} K_{1}^{(w)}\left(T^{\prime}\left(R, t^{\prime}\right)\right) P\left(R, t^{\prime}\right) R^{2} d R\right) d t^{\prime} \\
+\frac{2}{R_{2}^{2}-R_{1}^{2}} \int_{0}^{t} K_{2}^{\prime}\left(t, t^{\prime}\right)\left|\Omega_{r}\left(t^{\prime}\right)\right| \\
\cdot\left(\int_{R_{1}}^{R_{2}} K_{2}^{(w)}\left(T^{\prime}\left(R, t^{\prime}\right)\right) P\left(R, t^{\prime}\right) R^{2} d R\right) d t^{\prime} \\
+\frac{k Q(t)}{\pi\left(R_{2}^{2}-R_{1}^{2}\right)} .
\end{gathered}
$$

The friction torque transmitted by the clutch with friction coefficient $\mu\left(\Omega_{r}(t), R, T^{\prime}(R, t)\right)$ between friction linings is computed as a sum of basic torques integrating all over the entire contact surface of linings, and it reads

$$
M_{\mathrm{fr}}(t)=2 \pi \int_{R_{1}}^{R_{2}} \mu\left(\Omega_{r}(t), R, T^{\prime}(R, t)\right) R^{2} P(R, t) d R .
$$

4.3. Analytical Investigations. Suppose that in the initial time instant $t=0$ we take $U_{1}^{(w)}(R, 0)=0$ and $U_{2}^{(w)}(R, 0)=0$. Then, according to (9)

$$
E(0)=U^{(1)}(R, 0)+U^{(2)}(R, 0)=k P(R, 0)
$$

does not depend on $R$, and in consequence $P(R, 0)$ does not depend on $R$ either. Taking into account this observation and (14) one obtains

$$
\begin{aligned}
Q(0) & =2 \pi \int_{R_{1}}^{R_{2}} R P(R, 0) d R, \\
& \Longrightarrow Q(0)=2 \pi P(R, 0) \frac{R_{2}^{2}-R_{1}^{2}}{2} .
\end{aligned}
$$

On the basis of (21) and (22), we have

$$
P(R, 0)=\frac{Q(0)}{\pi\left(R_{2}^{2}-R_{1}^{2}\right)}, \quad E(0)=\frac{k Q(0)}{\pi\left(R_{2}^{2}-R_{1}^{2}\right)} .
$$

According to relation (20) for $\mu\left(\Omega_{r}(0), R, T^{\prime}(R, 0)\right)=\mu=$ constant the friction torque transmitted by the clutch in the initial moment $t=0$ is as follows:

$$
M_{\mathrm{fr}}(0)=\frac{2}{3} \mu Q(0) \frac{R_{2}^{3}-R_{1}^{3}}{R_{2}^{2}-R_{1}^{2}} .
$$

Further analysis of both differential and integral wear models requires the following simplifications: $Q(t)=Q=$ constant, $\Omega_{r}(t)=\Omega_{r}=$ constant, $\alpha_{1}=\alpha_{2}=1, \beta_{1}=\beta_{2}=1$, $K_{1}^{\prime}\left(t, t^{\prime}\right)=1, K_{2}^{\prime}\left(t, t^{\prime}\right)=1, K_{1}^{(w)}\left(T^{\prime}(R, t)\right)=K_{1}^{(w)}=$ constant, $K_{2}^{(w)}\left(T^{\prime}(R, t)\right)=K_{2}^{(w)}=$ constant, and $K_{1}^{(w)}+K_{2}^{(w)}=$ $K^{(w)}$. Taking into account assumptions $K_{1}^{\prime}\left(t, t^{\prime}\right)=1$ and $K_{2}^{\prime}\left(t, t^{\prime}\right)=1$, the equations describing wear with respect to the integral wear model can be written in a differential form which agrees with the differential wear model for $\alpha_{1}=\alpha_{2}=1$ and $\beta_{1}=\beta_{2}=1$. Thus, we obtain an analogous relation presented below, similar to the special case for the differential wear model. The introduced assumptions imply linear wear models, first written in the differential form and second in the integral form.

Owing to (7), wears in the initial time instant are governed by the following relations:

$$
\begin{aligned}
& \frac{\partial U_{1}^{(w)}(R, 0)}{\partial t}=\frac{K_{1}^{(w)} Q\left|\Omega_{r}\right|}{\pi\left(R_{2}^{2}-R_{1}^{2}\right)} R, \\
& \frac{\partial U_{2}^{(w)}(R, 0)}{\partial t}=\frac{K_{2}^{(w)} Q\left|\Omega_{r}\right|}{\pi\left(R_{2}^{2}-R_{1}^{2}\right)} R .
\end{aligned}
$$

Observe that speeds of wearing of friction linings $\partial U_{1}^{(w)}(R$, $0) / \partial t$ and $\partial U_{2}^{(w)}(R, 0) / \partial t$ are proportional to radius $R$ in the initial moment at each point of contact. The total speed of wearing of friction linings $\partial U^{(w)}(R, 0) / \partial t=\partial U_{1}^{(w)}(R, 0) / \partial t+$ $\partial U_{2}^{(w)}(R, 0) / \partial t$ has the form

$$
\frac{\partial U^{(w)}(R, 0)}{\partial t}=\frac{K^{(w)} Q\left|\Omega_{r}\right|}{\pi\left(R_{2}^{2}-R_{1}^{2}\right)} R .
$$

Equation (15) yields the speed of decrease of the distance between clutch shields in the initial time instant

$$
\frac{d E(0)}{d t}=\frac{2 K^{(w)} Q\left|\Omega_{r}\right|\left(R_{2}^{3}-R_{1}^{3}\right)}{3 \pi\left(R_{2}^{2}-R_{1}^{2}\right)^{2}} .
$$

Below, we show the way of solving equations governing contact pressures. Taking the above assumptions into (17) we obtain

$$
\begin{aligned}
K^{(w)} & \left|\Omega_{r}\right| R P(R, t)+k \frac{\partial P(R, t)}{\partial t} \\
& =\frac{2 K^{(w)}\left|\Omega_{r}\right|}{R_{2}^{2}-R_{1}^{2}} \int_{R_{1}}^{R_{2}} R^{2} P(R, t) d R .
\end{aligned}
$$

Applying the Laplace transformation to (28), we get

$$
\begin{gathered}
K^{(w)}\left|\Omega_{r}\right| R \bar{P}(R, s)+k s \bar{P}(R, s)-k P(R, 0) \\
=\frac{2 K^{(w)}\left|\Omega_{r}\right|}{R_{2}^{2}-R_{1}^{2}} \int_{R_{1}}^{R_{2}} R^{2} \bar{P}(R, s) d R .
\end{gathered}
$$

Direct application of (23) governing the contact pressure $P(R, 0)$ for a constant force $Q(t)=Q$ yields equation

$$
\begin{aligned}
\bar{P}(R, s)= & \left(K^{(w)}\left|\Omega_{r}\right| R+k s\right)^{-1} \\
& \cdot\left(\frac{2 K^{(w)}\left|\Omega_{r}\right|}{R_{2}^{2}-R_{1}^{2}} \int_{R_{1}}^{R_{2}} R^{2} \bar{P}(R, s) d R+\frac{k Q}{\pi\left(R_{2}^{2}-R_{1}^{2}\right)}\right),
\end{aligned}
$$

since

$$
P(R, \infty)=\lim _{s \rightarrow 0} s \bar{P}(R, s)
$$


and after next transformations of (30), the following relationship is derived:

$$
P(R, \infty)=\frac{1}{R}\left(\frac{2}{R_{2}^{2}-R_{1}^{2}} \int_{R_{1}}^{R_{2}} R^{2} P(R, \infty) d R\right) .
$$

In what follows, we are going to show that integral equation (32) has the following form:

$$
P(R, \infty)=\frac{A}{R} .
$$

In the beginning we prove that (33) is a solution to (32):

$$
\begin{aligned}
\frac{1}{R}\left(\frac{2}{R_{2}^{2}-R_{1}^{2}} \int_{R_{1}}^{R_{2}} R^{2} \frac{A}{R} d R\right) & =\frac{1}{R}\left(\frac{2}{R_{2}^{2}-R_{1}^{2}} A \int_{R_{1}}^{R_{2}} R d R\right) \\
& =\frac{A}{R} .
\end{aligned}
$$

Now our aim is to determine constant $A$. Substituting relation (33) into (14) and taking into account $t \rightarrow \infty$, the following constant $A$ is obtained

$$
A=\frac{Q}{2 \pi\left(R_{2}-R_{1}\right)} .
$$

Finally, in a steady state, we get contact pressure distribution $P(R, \infty)$ in the form

$$
P(R, \infty)=\frac{Q}{2 \pi\left(R_{2}-R_{1}\right)} \frac{1}{R} .
$$

The obtained contact pressure distributions in the steady state in the form of (36) allow us to determine the remaining relations. The speed of wearing of frictional clutch linings $\partial U_{1}^{(w)}(R, \infty) / \partial t$ and $\partial U_{2}^{(w)}(R, \infty) / \partial t$ in the steady state is as follows:

$$
\begin{aligned}
& \frac{\partial U_{1}^{(w)}(R, \infty)}{\partial t}=\frac{K_{1}^{(w)} Q\left|\Omega_{r}\right|}{2 \pi\left(R_{2}-R_{1}\right)}, \\
& \frac{\partial U_{2}^{(w)}(R, \infty)}{\partial t}=\frac{K_{2}^{(w)} Q\left|\Omega_{r}\right|}{2 \pi\left(R_{2}-R_{1}\right)} .
\end{aligned}
$$

It is constant on the entire contact surface (it does not depend on radius $R$ ). For this reason wears of linings $U_{1}^{(w)}(R, \infty)$ and $U_{2}^{(w)}(R, \infty)$ in each point of contact surface theoretically increase to infinity with constant speed of wear. The total speed of wear $\partial U^{(w)}(R, \infty) / \partial t\left(\partial U_{1}^{(w)}(R, \infty) / \partial t+\right.$ $\left.\partial U_{2}^{(w)}(R, \infty) / \partial t\right)$ takes the form

$$
\frac{\partial U^{(w)}(R, \infty)}{\partial t}=\frac{K^{(w)} Q\left|\Omega_{r}\right|}{2 \pi\left(R_{2}-R_{1}\right)} .
$$

In turn, the speed of decrease of the distance between clutch shields in the steady state is

$$
\frac{d E(\infty)}{d t}=\frac{K_{1}^{(w)} \mathrm{Q}\left|\Omega_{r}\right|}{2 \pi\left(R_{2}-R_{1}\right)}+\frac{K_{2}^{(w)} \mathrm{Q}\left|\Omega_{r}\right|}{2 \pi\left(R_{2}-R_{1}\right)}=\frac{K^{(w)} \mathrm{Q}\left|\Omega_{r}\right|}{2 \pi\left(R_{2}-R_{1}\right)}
$$

and is equal to the total speed of wear $\partial U^{(w)}(R, \infty) / \partial t$, and for this reason theoretically $E(\infty) \rightarrow \infty$. Friction torque transmitted by the clutch for $\mu=$ constant in the steady state $(t=\infty)$ is as follows:

$$
M_{\mathrm{fr}}(\infty)=\mu Q \frac{R_{1}+R_{2}}{2} .
$$

The above theoretical analysis allows us to appoint contact pressure distribution $P(R, 0)$, distance between clutch shields $E(0)$, and friction torque $M_{\mathrm{fr}}(0)$ transmitted by the clutch in the initial moment $(t=0)$. Initial values of wear at each point of the contact of friction linings are equal to zero. Besides, we obtain the speed of decrease of the distance between clutch shields $d E(0) / d t$ in the initial moment $(t=$ $0)$. After accepting additional simplifications, for the linear wear model we also estimated contact pressure distribution $P(R, \infty)$, the speed of decrease of the distance between clutch shields $d E(\infty) / d t$, and friction torque transmitted by the clutch $M_{\mathrm{fr}}(\infty)$ in the steady state $(t \rightarrow \infty)$.

4.4. Nondimensional Form. The obtained relations were also written in a nondimensional form. We introduce the similarity coefficients $t_{*}, U_{*}, T_{*}, \Omega_{*}, P_{*}, Q_{*}, M_{*}$, nondimensional times $\tau=t / t_{*}$ and $\xi=t^{\prime} / t_{*}$, nondimensional radius $r=\left(R-R_{1}\right) /\left(R_{2}-R_{1}\right)$, nondimensional geometrical parameter $\rho=R_{1} /\left(R_{2}-R_{1}\right)$, other nondimensional parameters $U_{*} / k P_{*}=l_{1}, Q_{*}(1+\rho)^{2} / \pi P_{*} R_{2}^{2}(1+2 \rho)=l_{2}, 2 \pi P_{*} R_{2}^{3} / M_{*}(1+$ $\rho)^{3}=k_{\text {fr }}$, the nondimensional functions

$$
\begin{gathered}
\frac{U_{1}^{(w)}\left(\left(R_{2}-R_{1}\right)(r+\rho), t_{*} \tau\right)}{U_{*}}=u_{1}^{(w)}(r, \tau), \\
\frac{U_{2}^{(w)}\left(\left(R_{2}-R_{1}\right)(r+\rho), t_{*} \tau\right)}{U_{*}}=u_{2}^{(w)}(r, \tau), \\
\frac{T^{\prime}\left(\left(R_{2}-R_{1}\right)(r+\rho), t_{*} \tau\right)}{T_{*}}=T(r, \tau), \\
\frac{\Omega_{r}\left(t_{*} \tau\right)}{\Omega_{*}}=\omega_{r}(\tau), \quad \frac{Q\left(t_{*} \tau\right)}{Q_{*}}=q(\tau), \\
\frac{P\left(\left(R_{2}-R_{1}\right)(r+\rho), t_{*} \tau\right)}{P_{*}}=p(r, \tau), \\
K_{1}^{\prime}\left(t_{*} \tau, t_{*} \xi\right)=K_{1}(\tau, \xi), \quad K_{2}^{\prime}\left(t_{*} \tau, t_{*} \xi\right)=K_{2}(\tau, \xi), \\
U_{*}=\eta(\tau), \quad \frac{M_{\mathrm{fr}}\left(t_{*} \tau\right)}{M_{*}}=F_{\mathrm{fr}}(\tau), \\
\Omega_{*} \frac{\Omega_{r}\left(t_{*} \tau\right)}{\Omega_{*},\left(R_{2}-R_{1}\right)(r+\rho),} \\
\left.T_{*} \frac{T^{\prime}\left(\left(R_{2}-R_{1}\right)(r+\rho), t_{*} \tau\right)}{T_{*}}\right)
\end{gathered}
$$


the nondimensional wear coefficients for the differential wear model

$$
\begin{aligned}
& \frac{t_{*} \Omega_{*}^{\beta_{1}} P_{*}^{\alpha_{1}} R_{2}^{\beta_{1}}}{U_{*}(1+\rho)^{\beta_{1}}} K_{1}^{(w)}\left(T_{*} \frac{T^{\prime}\left(\left(R_{2}-R_{1}\right)(r+\rho), t_{*} \tau\right)}{T_{*}}\right) \\
& \quad=k_{1}^{(w)}(T(r, \tau)), \\
& \frac{t_{*} \Omega_{*}^{\beta_{2}} P_{*}^{\alpha_{2}} R_{2}^{\beta_{2}}}{U_{*}(1+\rho)^{\beta_{2}}} K_{2}^{(w)}\left(T_{*} \frac{T^{\prime}\left(\left(R_{2}-R_{1}\right)(r+\rho), t_{*} \tau\right)}{T_{*}}\right) \\
& \quad=k_{2}^{(w)}(T(r, \tau)),
\end{aligned}
$$

and the nondimensional wear coefficients for the integral wear model

$$
\begin{aligned}
& \frac{t_{*} \Omega_{*} P_{*} R_{2}}{U_{*}(1+\rho)} K_{1}^{(w)}\left(T_{*} \frac{T^{\prime}\left(\left(R_{2}-R_{1}\right)(r+\rho), t_{*} \tau\right)}{T_{*}}\right) \\
& \quad=k_{1}^{(w)}(T(r, \tau)) \\
& \frac{t_{*} \Omega_{*} P_{*} R_{2}}{U_{*}(1+\rho)} K_{2}^{(w)}\left(T_{*} \frac{T^{\prime}\left(\left(R_{2}-R_{1}\right)(r+\rho), t_{*} \tau\right)}{T_{*}}\right) \\
& \quad=k_{2}^{(w)}(T(r, \tau)) .
\end{aligned}
$$

Then, owing to (7), the wear of individual friction linings has the following nondimensional form (differential wear model):

$$
\begin{aligned}
& \frac{\partial u_{1}^{(w)}(r, \tau)}{\partial \tau}=k_{1}^{(w)}(T(r, \tau))\left|\omega_{r}(\tau)\right|^{\beta_{1}}(r+\rho)^{\beta_{1}} p^{\alpha_{1}}(r, \tau), \\
& \frac{\partial u_{2}^{(w)}(r, \tau)}{\partial \tau}=k_{2}^{(w)}(T(r, \tau))\left|\omega_{r}(\tau)\right|^{\beta_{2}}(r+\rho)^{\beta_{2}} p^{\alpha_{2}}(r, \tau),
\end{aligned}
$$

whereas on the basis of (8) the wear of individual friction linings has the following nondimensional form (integral wear model):

$$
\begin{aligned}
& u_{1}^{(w)}(r, \tau) \\
& =(r+\rho) \int_{0}^{\tau} k_{1}^{(w)}(T(r, \xi))\left|\omega_{r}(\xi)\right| K_{1}(\tau, \xi) p(r, \xi) d \xi, \\
& u_{2}^{(w)}(r, \tau) \\
& =(r+\rho) \int_{0}^{\tau} k_{2}^{(w)}(T(r, \xi))\left|\omega_{r}(\xi)\right| K_{2}(\tau, \xi) p(r, \xi) d \xi .
\end{aligned}
$$

Nondimensional equations (15) and (16) describing a decrease of the distance between clutch shields are (for the differential and integral wear model, resp.)

$$
\begin{aligned}
\frac{d \eta(\tau)}{d \tau}= & \frac{2}{1+2 \rho}\left|\omega_{r}(\tau)\right|^{\beta_{1}} \\
& \cdot \int_{0}^{1} k_{1}^{(w)}(T(r, \tau))(r+\rho)^{1+\beta_{1}} p^{\alpha_{1}}(r, \tau) d r \\
& +\frac{2}{1+2 \rho}\left|\omega_{r}(\tau)\right|^{\beta_{2}} \\
& \cdot \int_{0}^{1} k_{2}^{(w)}(T(r, \tau))(r+\rho)^{1+\beta_{2}} p^{\alpha_{2}}(r, \tau) d r \\
& +\frac{l_{2}}{l_{1}} \frac{d q(\tau)}{d \tau}, \\
\eta(\tau) & \frac{2}{1+2 \rho} \int_{0}^{\tau} K_{1}(\tau, \xi)\left|\omega_{r}(\xi)\right| \\
& \cdot\left(\int_{0}^{1} k_{1}^{(w)}(T(r, \xi)) p(r, \xi)\right. \\
& +\frac{l_{2}}{l_{1}} q(\tau) \cdot \\
& +\frac{2}{1+2 \rho} \int_{0}^{\tau} K_{2}(\tau, \xi)\left|\omega_{r}(\xi)\right| \\
& \cdot\left(\int_{0}^{1} k_{2}^{(w)}(T(r, \xi)) p(r, \xi)\right. \\
& \left.\cdot(r+\rho)^{2} d r\right) d \xi \\
& \\
& \\
& \\
& \\
& \\
& \\
& \\
&
\end{aligned}
$$

Equations (17) and (19) yield the following nondimensional relationship (differential wear model):

$$
\begin{aligned}
l_{1} k_{1}^{(w)}(T(r, \tau))\left|\omega_{r}(\tau)\right|^{\beta_{1}}(r+\rho)^{\beta_{1}} p^{\alpha_{1}}(r, \tau) & \\
+ & l_{1} k_{2}^{(w)}(T(r, \tau))\left|\omega_{r}(\tau)\right|^{\beta_{2}} \\
& \cdot(r+\rho)^{\beta_{2}} p^{\alpha_{2}}(r, \tau)+\frac{\partial p(r, \tau)}{\partial \tau} \\
= & \frac{2 l_{1}}{1+2 \rho}\left|\omega_{r}(\tau)\right|^{\beta_{1}} \\
& \cdot \int_{0}^{1} k_{1}^{(w)}(T(r, \tau))(r+\rho)^{1+\beta_{1}} p^{\alpha_{1}}(r, \tau) d r
\end{aligned}
$$




$$
\begin{aligned}
&+\frac{2 l_{1}}{1+2 \rho}\left|\omega_{r}(\tau)\right|^{\beta_{2}} \\
& \cdot \int_{0}^{1} k_{2}^{(w)}(T(r, \tau))(r+\rho)^{1+\beta_{2}} p^{\alpha_{2}}(r, \tau) d r+l_{2} \frac{d q(\tau)}{d \tau} \\
& l_{1}(r+\rho) \int_{0}^{\tau} k_{1}^{(w)}(T(r, \xi))\left|\omega_{r}(\xi)\right| K_{1}(\tau, \xi) p(r, \xi) d \xi \\
&+l_{1}(r+\rho) \int_{0}^{\tau} k_{2}^{(w)}(T(r, \xi))\left|\omega_{r}(\xi)\right| K_{2}(\tau, \xi) \\
&=\frac{2 l_{1}}{1+2 \rho} \int_{0}^{\tau} K_{1}(\tau, \xi)\left|\omega_{r}(\xi)\right| \\
& \quad \cdot\left(\int_{0}^{1} k_{1}^{(w)}(T(r, \xi)) p(r, \xi)(r+\rho)^{2} d r\right) d \xi \\
& \quad+\frac{2 l_{1}}{1+2 \rho} \int_{0}^{\tau} K_{2}(\tau, \xi)\left|\omega_{r}(\xi)\right| \\
& \quad \cdot\left(\int_{0}^{1} k_{2}^{(w)}(T(r, \xi)) p(r, \xi)(r+\rho)^{2} d r\right) d \xi \\
&+l_{2} q(\tau)
\end{aligned}
$$

for the integral wear model. On the basis of (20), we get the associated nondimensional relation describing friction torque transmitted by the clutch

$$
F_{\mathrm{fr}}(\tau)=k_{\mathrm{fr}} \int_{0}^{1} f\left(\omega_{r}(\tau), r, T(r, \tau)\right)(r+\rho)^{2} p(r, \tau) d r .
$$

For further analysis we assume that $\Omega_{*}=\Omega_{r}, t_{*}=$ $1 / \Omega_{*}, U_{*}=E(0), P_{*}=P(R, 0), Q_{*}=Q, M_{*}=M_{\mathrm{fr}}(0)$. Hence, we obtain $l_{1}=1$ and $l_{2}=1$. Since the friction torque transmitted by the clutch is normalized (referring to the initial value), the value of coefficient $k_{\mathrm{fr}}$ is irrelevant (it should be positive only). The above presented assumption yields the following functions: $\omega_{r}=1, q=1, p(r, 0)=$ $1, \eta(0)=1$, and $F_{\mathrm{fr}}(0)=1$. The initial wear of frictional linings in nondimensional form is also equal to zero, namely, $u_{1}^{(w)}(r, 0)=0$ and $u_{2}^{(w)}(r, 0)=0$. In addition, we take the following assumptions: $\alpha_{1}=\alpha_{2}=1, \beta_{1}=\beta_{2}=$ $1, K_{1}(\tau, \xi)=1, K_{2}(\tau, \xi)=1, k_{1}^{(w)}(T(r, \tau))=k_{1}^{(w)}=$ constant, $k_{2}^{(w)}(T(r, \tau))=k_{2}^{(w)}=$ constant, and $k_{1}^{(w)}+k_{2}^{(w)}=$ $k^{(w)}$. Then, initial speeds of wearing of linings have the following nondimensional form,

$$
\begin{aligned}
& \frac{\partial u_{1}^{(w)}(r, 0)}{\partial \tau}=k_{1}^{(w)}\left|\omega_{r}\right|(r+\rho) p(r, 0)=k_{1}^{(w)}(r+\rho), \\
& \frac{\partial u_{2}^{(w)}(r, 0)}{\partial \tau}=k_{2}^{(w)}\left|\omega_{r}\right|(r+\rho) p(r, 0)=k_{2}^{(w)}(r+\rho),
\end{aligned}
$$

and are the linear functions of the nondimensional radius $r$. The nondimensional total speed of wearing of linings in the initial moment is

$$
\frac{\partial u^{(w)}(r, 0)}{\partial \tau}=k^{(w)}\left|\omega_{r}\right|(r+\rho) p(r, 0)=k^{(w)}(r+\rho),
$$

and it is proportional to the nondimensional radius $r$. The speed of decrease of the distance between clutch shields (in the initial time moment) is simplified to the following nondimensional form:

$$
\frac{d \eta(0)}{d \tau}=k^{(w)} \frac{2}{3} \frac{(1+\rho)^{2}+\rho(1+\rho)+\rho^{2}}{1+2 \rho} .
$$

Contact pressure distribution in the steady state is

$$
p(r, \infty)=\left(\frac{1}{2}+\rho\right) \frac{1}{r+\rho},
$$

whereas nondimensional speeds of wearing of clutch linings in the steady state are

$$
\begin{aligned}
& \frac{\partial u_{1}^{(w)}(r, \infty)}{\partial \tau}=\left(\frac{1}{2}+\rho\right) k_{1}^{(w)}, \\
& \frac{\partial u_{2}^{(w)}(r, \infty)}{\partial \tau}=\left(\frac{1}{2}+\rho\right) k_{2}^{(w)},
\end{aligned}
$$

and the total speed of wearing of clutch linings in the steady state is simplified to the following nondimensional form:

$$
\frac{\partial u^{(w)}(r, \infty)}{\partial \tau}=\left(\frac{1}{2}+\rho\right) k^{(w)} .
$$

Nondimensional speed of decrease of the distance between clutch shields in the steady state is as follows:

$$
\frac{d \eta(\infty)}{d \tau}=\left(\frac{1}{2}+\rho\right) k_{1}^{(w)}+\left(\frac{1}{2}+\rho\right) k_{2}^{(w)}=\left(\frac{1}{2}+\rho\right) k^{(w)},
$$

and it is equal to the total speed of wearing of clutch linings. For this reason for $\tau \rightarrow \infty$ theoretically also $\eta(\tau) \rightarrow \infty$. Moreover, nondimensional friction torque transmitted by the clutch in the steady state is described by the relation

$$
F_{\mathrm{fr}}(\infty)=\frac{3}{4} \frac{(1+2 \rho)^{2}}{(1+\rho)^{2}+\rho(1+\rho)+\rho^{2}} .
$$

4.5. Numerical Computational Methods. Integrals occurring in nondimensional differential, integral, and integrodifferential equations are written using the trapezia method. ODEs obtained in this way were solved using the fourth-order Runge-Kutta method. In order to solve (44)-(50), we divided nondimensional radius $r$ on $m$ even segments taking $\Delta_{r}=$ $1 / m, r_{i}=\Delta_{r} i, r_{j}=\Delta_{r} j$. Radius-dependent integrals in (46)-(50) are replaced with the sum using the method of trapezia (rates of the method are $a_{0}=a_{m}=1 / 2, a_{j}=1$, $j=1,2, \ldots, m-1)$. Time-dependent integrals in (45), (47), 
and (49) are also replaced with the sum using the method of trapezia (rates of the method are $b_{0}=b_{n}=1 / 2, b_{j}=$ $1, j=1,2, \ldots, n-1)$. In addition, we adopted the following indications: $p\left(r_{i}, \tau\right)=p_{i}(\tau), T\left(r_{i}, \tau\right)=T_{i}(\tau), u_{1}^{(w)}\left(r_{i}, \tau\right)=$ $u_{1, i}^{(w)}(\tau)$, and $u_{2}^{(w)}\left(r_{i}, \tau\right)=u_{2, i}^{(w)}(\tau)$. For time $\tau$ on the basis of (44), wears of individual friction linings are

$$
\begin{aligned}
& \frac{d u_{1, i}^{(w)}(\tau)}{d \tau}=k_{1}^{(w)}\left(T_{i}(\tau)\right)\left|\omega_{r}(\tau)\right|^{\beta_{1}}\left(r_{i}+\rho\right)^{\beta_{1}} p_{i}^{\alpha_{1}}(\tau), \\
& \frac{d u_{2, i}^{(w)}(\tau)}{d \tau}=k_{2}^{(w)}\left(T_{i}(\tau)\right)\left|\omega_{r}(\tau)\right|^{\beta_{2}}\left(r_{i}+\rho\right)^{\beta_{2}} p_{i}^{\alpha_{2}}(\tau),
\end{aligned}
$$

for the differential wear model, and for the integral wear model we have

$$
\begin{aligned}
u_{1, i}^{(w)}\left(\tau_{n}\right)= & \left(r_{i}+\rho\right) \Delta_{\tau} \\
& \cdot \sum_{j=0}^{n} b_{j} k_{1}^{(w)}\left(T_{i}\left(\tau_{j}\right)\right)\left|\omega_{r}\left(\tau_{j}\right)\right| K_{1}\left(\tau_{n}, \tau_{j}\right) p_{i}\left(\tau_{j}\right), \\
u_{2, i}^{(w)}\left(\tau_{n}\right)= & \left(r_{i}+\rho\right) \Delta_{\tau} \\
& \cdot \sum_{j=0}^{n} b_{j} k_{2}^{(w)}\left(T_{i}\left(\tau_{j}\right)\right)\left|\omega_{r}\left(\tau_{j}\right)\right| K_{2}\left(\tau_{n}, \tau_{j}\right) p_{i}\left(\tau_{j}\right) .
\end{aligned}
$$

On the basis of (46) we get (for the differential wear model)

$$
\begin{aligned}
\frac{d \eta(\tau)}{d \tau}= & \frac{2}{1+2 \rho}\left|\omega_{r}(\tau)\right|^{\beta_{1}} \Delta_{r} \\
& \cdot \sum_{i=0}^{m} a_{i} k_{1}^{(w)}\left(T_{i}(\tau)\right)\left(r_{i}+\rho\right)^{1+\beta_{1}} p_{i}^{\alpha_{1}}(\tau) \\
& +\frac{2}{1+2 \rho}\left|\omega_{r}(\tau)\right|^{\beta_{2}} \Delta_{r} \\
& \cdot \sum_{i=0}^{m} a_{i} k_{2}^{(w)}\left(T_{i}(\tau)\right)\left(r_{i}+\rho\right)^{1+\beta_{2}} p_{i}^{\alpha_{2}}(\tau)+\frac{l_{2}}{l_{1}} \frac{d q(\tau)}{d \tau},
\end{aligned}
$$

whereas (47) yielded (for the integral wear model)

$$
\begin{aligned}
\eta\left(\tau_{n}\right)= & \frac{2}{1+2 \rho} \Delta_{\tau} \sum_{j=0}^{n} b_{j} K_{1}\left(\tau_{n}, \tau_{j}\right)\left|\omega_{r}\left(\tau_{j}\right)\right| \\
& \cdot\left(\Delta_{r} \sum_{i=0}^{m} a_{i} k_{1}^{(w)}\left(T_{i}\left(\tau_{j}\right)\right) p_{i}\left(\tau_{j}\right)\left(r_{i}+\rho\right)^{2}\right) \\
& +\frac{2}{1+2 \rho} \Delta_{\tau} \sum_{j=0}^{n} b_{j} K_{2}\left(\tau_{n}, \tau_{j}\right)\left|\omega_{r}\left(\tau_{j}\right)\right| \\
& \cdot\left(\Delta_{r} \sum_{i=0}^{m} a_{i} k_{2}^{(w)}\left(T_{i}\left(\tau_{j}\right)\right) p_{i}\left(\tau_{j}\right)\left(r_{i}+\rho\right)^{2}\right) \\
& +\frac{l_{2}}{l_{1}} q\left(\tau_{n}\right) .
\end{aligned}
$$

Using (48), the contact pressure is governed by (the differential wear model)

$$
\begin{aligned}
\frac{d p_{i}(\tau)}{d \tau}= & -l_{1} k_{1}^{(w)}\left(T_{i}(\tau)\right)\left|\omega_{r}(\tau)\right|^{\beta_{1}}\left(r_{i}+\rho\right)^{\beta_{1}} p_{i}^{\alpha_{1}}(\tau) \\
& -l_{1} k_{2}^{(w)}\left(T_{i}(\tau)\right)\left|\omega_{r}(\tau)\right|^{\beta_{2}}\left(r_{i}+\rho\right)^{\beta_{2}} p_{i}^{\alpha_{2}}(\tau) \\
& +\frac{2 l_{1}}{1+2 \rho}\left|\omega_{r}(\tau)\right|^{\beta_{1}} \Delta_{r} \\
& \cdot \sum_{j=0}^{m} a_{j} k_{1}^{(w)}\left(T_{j}(\tau)\right)\left(r_{j}+\rho\right)^{1+\beta_{1}} p_{j}^{\alpha_{1}}(\tau) \\
& +\frac{2 l_{1}}{1+2 \rho}\left|\omega_{r}(\tau)\right|^{\beta_{2}} \Delta_{r} \\
& \cdot \sum_{j=0}^{m} a_{j} k_{2}^{(w)}\left(T_{j}(\tau)\right)\left(r_{j}+\rho\right)^{1+\beta_{2}} p_{j}^{\alpha_{2}}(\tau)+l_{2} \frac{d q(\tau)}{d \tau} .
\end{aligned}
$$

On the other hand, (49) yielded the contact pressure equations (the integral wear model)

$$
\begin{array}{r}
p_{i}\left(\tau_{n}\right)\left(1+0.5 A_{i, n} k_{1}^{(w)}\left(T_{i}\left(\tau_{n}\right)\right)+0.5 A_{i, n} k_{2}^{(w)}\left(T_{i}\left(\tau_{n}\right)\right)\right) \\
-0.5 B_{n}\left(\sum_{i=0}^{m} a_{i} k_{1}^{(w)}\left(T_{i}\left(\tau_{n}\right)\right) p_{i}\left(\tau_{n}\right)\left(r_{i}+\rho\right)^{2}\right. \\
\left.+\sum_{i=0}^{m} a_{i} k_{2}^{(w)}\left(T_{i}\left(\tau_{n}\right)\right) p_{i}\left(\tau_{n}\right)\left(r_{i}+\rho\right)^{2}\right) \\
=B_{n} \sum_{j=0}^{n-1} b_{j} K_{1}\left(\tau_{n}, \tau_{j}\right)\left|\omega_{r}\left(\tau_{j}\right)\right| \\
\cdot\left(\sum_{i=0}^{m} a_{i} k_{1}^{(w)}\left(T_{i}\left(\tau_{j}\right)\right) p_{i}\left(\tau_{j}\right)\left(r_{i}+\rho\right)^{2}\right) \\
+B_{n} \sum_{j=0}^{n-1} b_{j} K_{2}\left(\tau_{n}, \tau_{j}\right)\left|\omega_{r}\left(\tau_{j}\right)\right| \\
\cdot\left(\sum_{i=0}^{m} a_{i} k_{2}^{(w)}\left(T_{i}\left(\tau_{j}\right)\right) p_{i}\left(\tau_{j}\right)\left(r_{i}+\rho\right)^{2}\right)+l_{2} q\left(\tau_{n}\right) \\
-A_{i, n}\left(k_{1}^{(w)}\left(T_{i}\left(\tau_{n}\right)\right) \sum_{j=0}^{n-1} b_{j} K_{1}\left(\tau_{n}, \tau_{j}\right) p_{i}\left(\tau_{j}\right)\right. \\
\left.+k_{2}^{(w)}\left(T_{i}\left(\tau_{n}\right)\right) \sum_{j=0}^{n-1} b_{j} K_{2}\left(\tau_{n}, \tau_{j}\right) p_{i}\left(\tau_{j}\right)\right),
\end{array}
$$


where $A_{i, n}=l_{1}\left|\omega_{r}\left(\tau_{n}\right)\right|\left(r_{i}+\rho\right) \Delta_{\tau}$ and $B_{n}=2 l_{1} \Delta_{r} \Delta_{\tau} /(1+2 \rho)$. Moreover, on the basis of (50) friction torque transmitted by the clutch is as follows:

$$
\begin{gathered}
F_{\mathrm{fr}}(\tau)=k_{\mathrm{fr}} \Delta_{r} \sum_{i=0}^{m} a_{i} f\left(\omega_{r}(\tau), r_{i}, T_{i}(\tau)\right)\left(r_{i}+\rho\right)^{2} p_{i}(\tau), \\
F_{\mathrm{fr}}\left(\tau_{n}\right)=k_{\mathrm{fr}} \Delta_{r} \sum_{i=0}^{m} a_{i} f\left(\omega_{r}\left(\tau_{n}\right), r_{i}, T_{i}\left(\tau_{n}\right)\right)\left(r_{i}+\rho\right)^{2} p_{i}\left(\tau_{n}\right) .
\end{gathered}
$$

Assuming again coefficients $\Omega_{*}=\Omega_{r}, t_{*}=1 / \Omega_{*}, U_{*}=$ $E(0), P_{*}=P(R, 0), Q_{*}=Q$ and $M_{*}=M_{\mathrm{fr}}(0)$, we have $l_{1}=1, l_{2}=1, \omega_{r}=1, q=1, p(r, 0)=1, \eta(0)=1$, and $F_{\text {fr }}(0)=1$.

In the above, the system of first-order ODEs is obtained, including $2(m+1)$ equations which describe the distributions of wear of individual clutch linings, one equation governing a decrease of the distance between clutch shields, and $m+1$ equations regarding the contact pressure distribution. In the case of integral wear model algebraic equations are obtained, including $2(m+1)$ equations for wear distributions, $m+1$ heterogeneous equations for contact pressure distribution, and one equation describing a decrease of the distance between clutch shields. Moreover, for both cases of wear models we obtain the equation describing friction torque transmitted by the clutch. The appropriate numerical algorithms were prepared in $\mathrm{C}++$ for numerical analysis for various values of system and numerical parameters. For numerical calculations presented in this paper we take $m=100$; however satisfactory results can be obtained also for $m=10$. The appropriate first-order ODEs are derived using the fourth-order RungeKutta method with constant time step $\Delta_{\tau}=0.01$, and the appropriate algebraic equations are obtained also by taking $\Delta_{\tau}=0.01$.

\section{Experimental Verification}

In this section we present the designed and built experimental stand, that is, a mechatronic system with friction clutch. It is used to verify and validate the applied mathematical models describing wear processes in the mechanical friction clutch.

5.1. Experimental Stand: A Mechatronic System with Friction Clutch. The presented experimental stand comprises a typical mechanical system with a friction clutch operating in a mechatronic system. Moreover, the experimental stand is equipped with an external power supply, measuring equipment, and a computer with appropriate software. Main parts of the considered experimental stand are presented in Figure 2. The stand consists of a driving part, driven part, and friction clutch which connects the driving and driven parts. The driving part (Figure 2(a)) includes asynchronous motor 1 controlled by a single-phase AC inverter 2 . The applied inverter is connected with a computer via the control/measurement module and USB port, which allows for control of the engine rotational speed through the applied computer program. To determine angular position of the active part of the clutch (motor) optical incremental encoder
3 is used. The differentiation of angular position with respect to time provides information on the motor angular velocity. The driven part (Figure 2(b)) includes DC motor 4 working as an electric generator which generates an appropriate antitorque depending on a connected load. Moreover, in the driven part of the system there is friction brake 5 and optical incremental encoder 6 (the same as in the driven part and playing a similar role). The friction torque transmitted by the clutch is measured by dynamic torque sensor 7. A member coupling both devices is the mechanical friction clutch (Figure 2(c)) consisting of clutch shields 8, friction linings 9, and spring 10 pressing the shields and controlled by an electronic system (not visible in the figure). A member coupling the mechanical system with computer software is a USB-4711A controller and measuring module 11 (Figure 2(d)) with analogue and digital inputs and outputs, connected with a computer via USB port 12 .

5.2. Research Methodology. To carry out experimental studies of wear processes occurring in the friction clutch the mechatronic system with friction clutch shown in Figure 2 was used. The sensor technology existing in the system (appropriate sensors coupled to a computer via a USB control and measurement module) provides a convenient way to read and then to process and archive the results. In order to conduct experiments regarding the wear of friction linings of the clutch shields a suitable construction of such a coupling to measure appropriate parameters describing these issues has been designed and built. Figure 2(c) shows the built clutch structure. This construction of coupling (as shown in Figure 2(c)) consists of three parts: active, passive, and connecting. The active and passive parts of the clutch, aluminum shields included, may be covered by linings using relevant friction materials. Clutch shields (and consequently also the friction linings) are pressed by a compression spring in the axial direction of the coupling force produced by the linear actuator located in the bottom (invisible part of the stand). Experimental investigations were compared with analytical solutions and numerical calculations.

Frictional linings used in the investigations are made of agglomerated cork which is a natural friction material used in friction clutches and brakes. This material was chosen for the following reasons: (a) it has a relatively high friction coefficient, so there is no need to apply large forces to press the shields in order to transfer large friction torque; (b) wear coefficient is relatively high and investigations of wear processes are possible in a relatively short period of time.

5.3. Investigations of Wear Processes. The experimental verification of wear processes of the clutch friction linings was carried out in the system shown in Figure 2. The measuring system consisted of two identical (attached to various parts of the clutch) shields with glued friction linings. Clutch shields were pressed by axial force $Q=$ constant via a spring which was compressed by the linear actuator located in the bottom part of the experimental stand. Passive part of the clutch was fixed to the stand, while the active one moved at a constant 


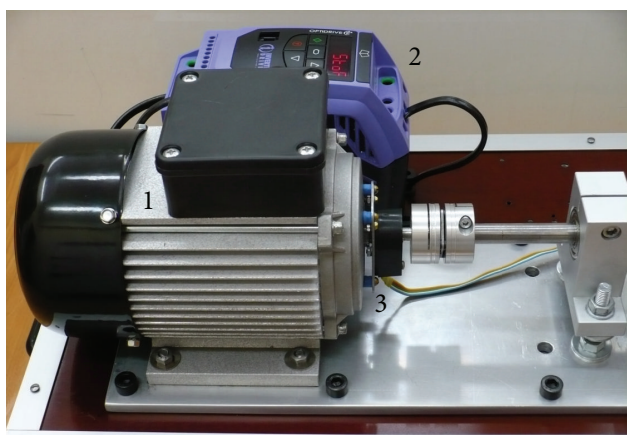

(a)

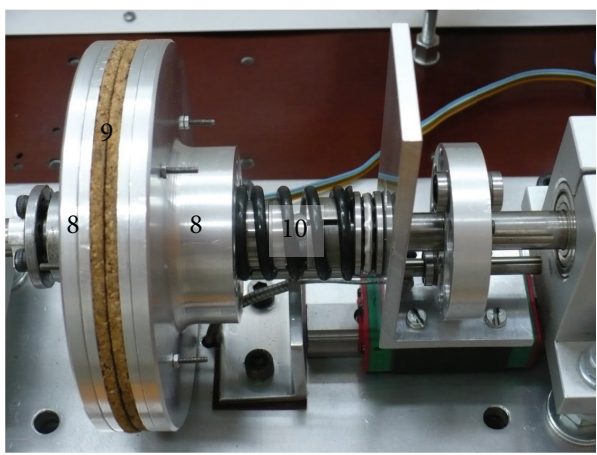

(c)

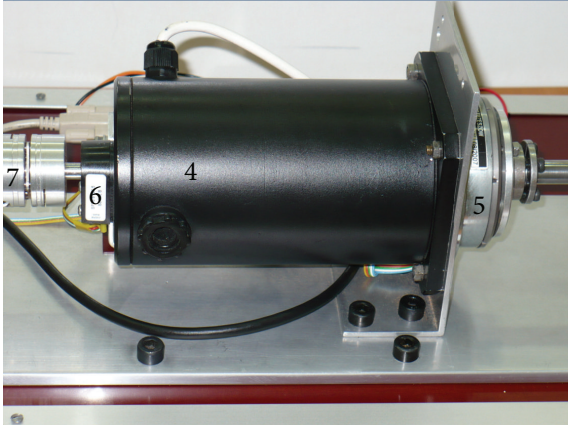

(b)

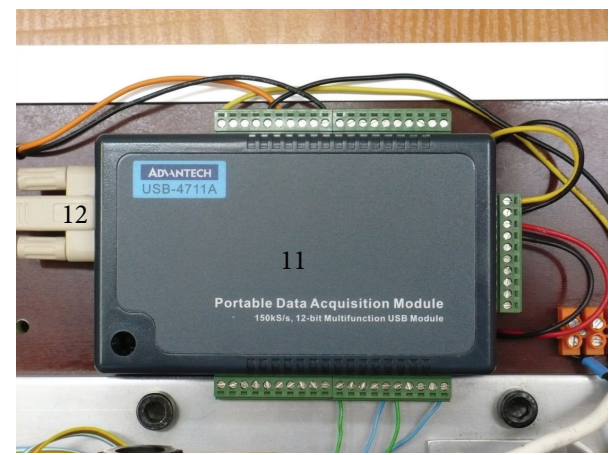

(d)

FIGURE 2: Main parts of the experimental stand: (a) driving part; (b) driven part; (c) regulated friction clutch; (d) control and measuring USB-4711 module.
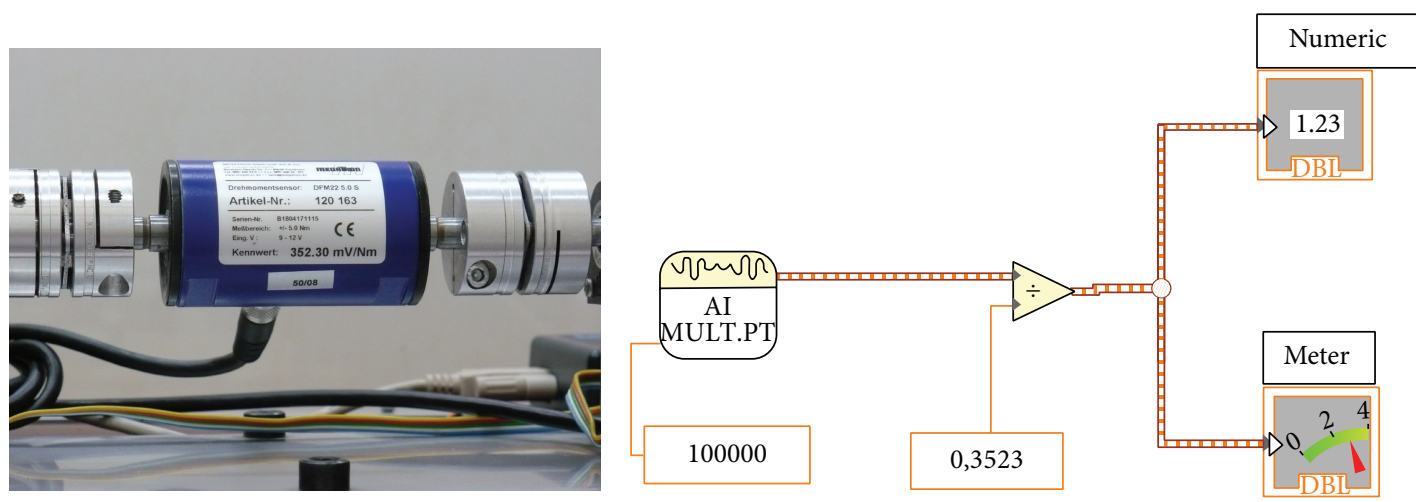

FIGURE 3: Torque sensor with the appropriate LabView software for processing electrical signal from the sensor.

angular velocity $\Omega_{r}=$ constant; therefore the relative sliding velocity of shields was also $\Omega_{r}$.

The experimental investigation of wear processes was carried out in stationary conditions (at constant force $Q$ pressing the shields and constant angular sliding velocity $\Omega_{r}$ ). Measurements were taken at regular time intervals $\Delta T$ after stopping the system. We measured a decrease of the distance between clutch shields, maximum static friction torque transmitted by the clutch and contact pressures in selected points of the contact surface of linings. A decrease of the distance between clutch shields $E(t)$ was measured using electronic precision calipers as the arithmetic mean of four measurements for various relative angular positions of the shields (after each measurement the shields were rotated relatively to each other by an angle equal to $\pi / 2$ ). Friction torque $M_{\mathrm{fr}}$ transmitted by the clutch was measured using a torque sensor located on the passive part of the clutch. The electrical signal including information about the values of the friction torque was processed by the control-measuring module and transmitted to the computer. Figure 3 shows the applied torque sensor connected with the USB-4711A module and diagram with connection of LabView elements, which converts the electrical signal from the sensor to the value of friction torque. Also in this case we assumed as the value of the transmitted friction torque the arithmetic average of four measurements for respective (differing by $\pi / 2$ ) relative 


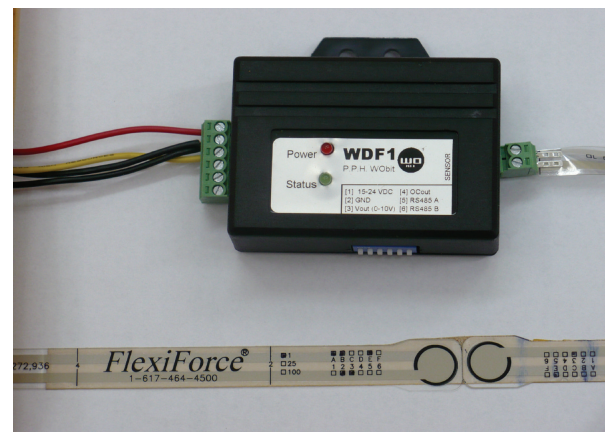

FIGURE 4: Contact pressure sensors with a corresponding transducer.

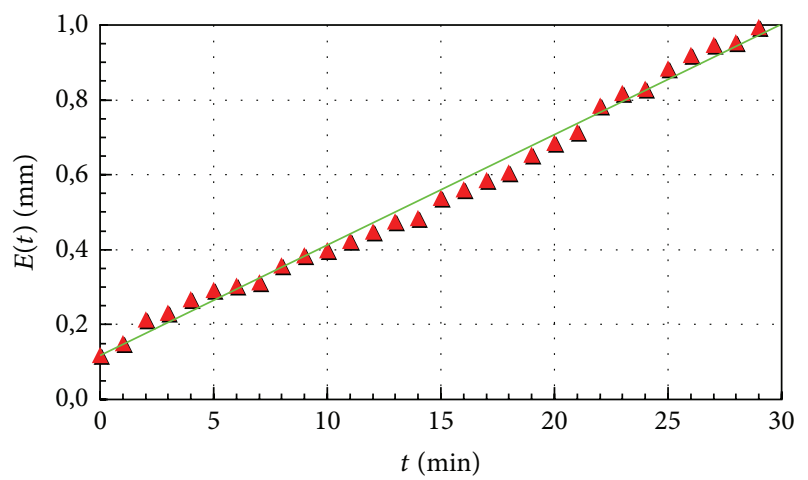

- Experimental data Linear approximation

FIGURE 5: Decrease of distance between clutch shields during wear processes.

angular positions of the clutch shields. For the initial time and after completion of the test, contact pressures $P(R, t)$ were also measured at the contact surface using a film pressure sensor located between the pressed clutch shields. Figure 4 shows the contact pressure sensors with a corresponding transducer. As before, the measurements were carried out for four various relative angular positions of the clutch shields, and a pressure value at a given point of contact surface was assumed as the arithmetic mean of the obtained values. The contact pressure sensor had the shape of a thin strip about $0.2 \mathrm{~cm}$ thick and approximately $1 \mathrm{~cm}$ wide. This sensor was placed along the diameter of the shields which were pressed with the same force as in the wear processes. While measuring power $Q$ of clamping, the sensor is sandwiched among the foil cladding. Therefore, the pressure value measured by the sensor was shown to be greater than the actual value occurring in the same conditions during the wear process. However, due to the proper placement of the contact pressure sensor along a diameter and a cladding of constant thickness over the entire length of the sensor, the values measured at the time of the contact pressure along the radius of the lining are proportional to the value observed during the wear process. Since the values measured at the time of contact pressure do not correspond to the values reported during the wear

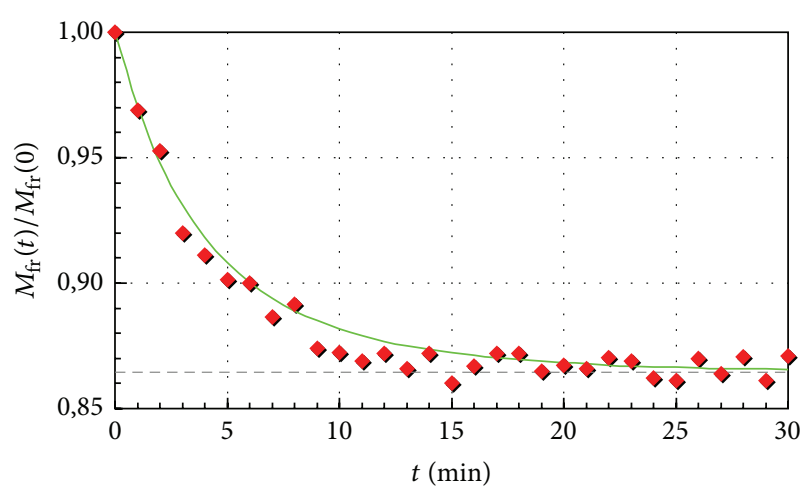

Experimental data Numerical results

FIGURE 6: Changes of friction torque transmitted by the clutch in time.

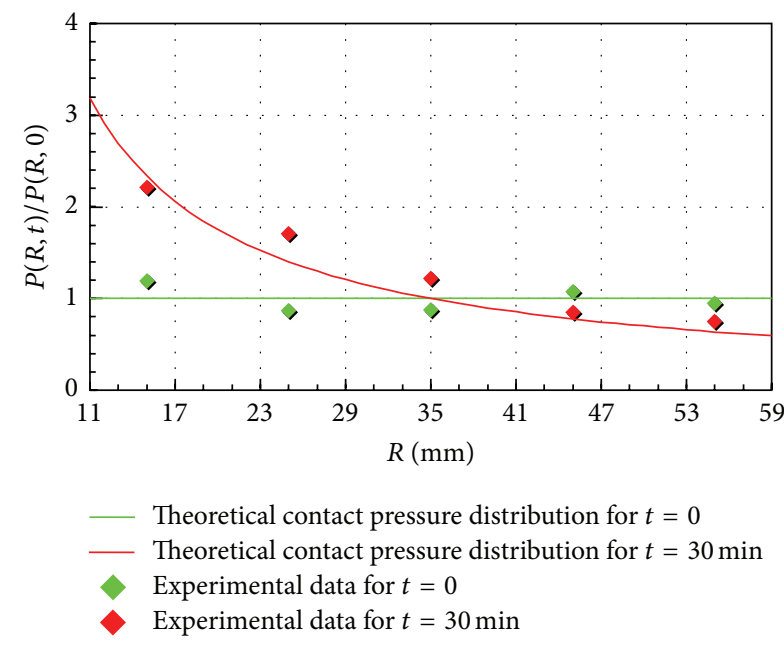

FIGURE 7: Contact pressure distributions obtained analytically and experimentally in the initial time and in the steady state.

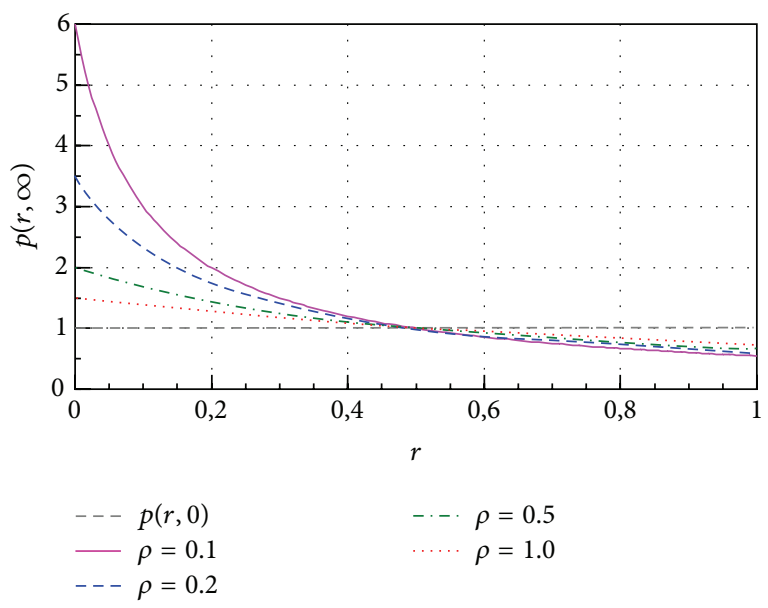

FIGURE 8: Contact pressure distributions in the steady state obtained analytically. 


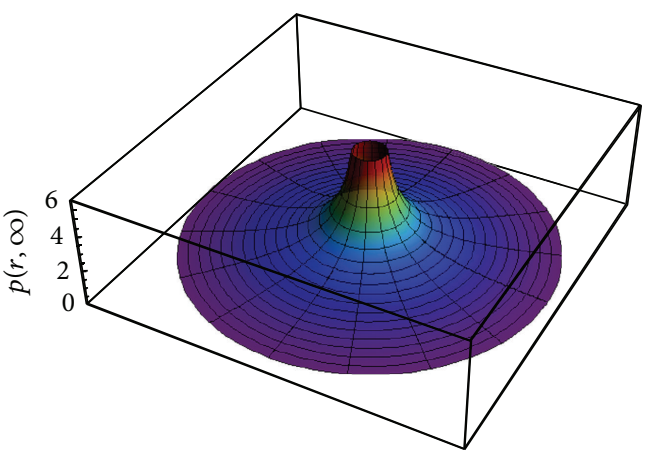

(a) $\rho=0.1$

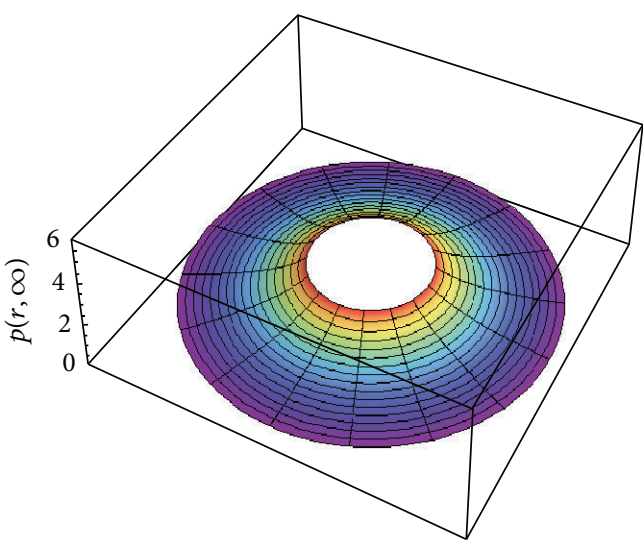

(c) $\rho=0.5$

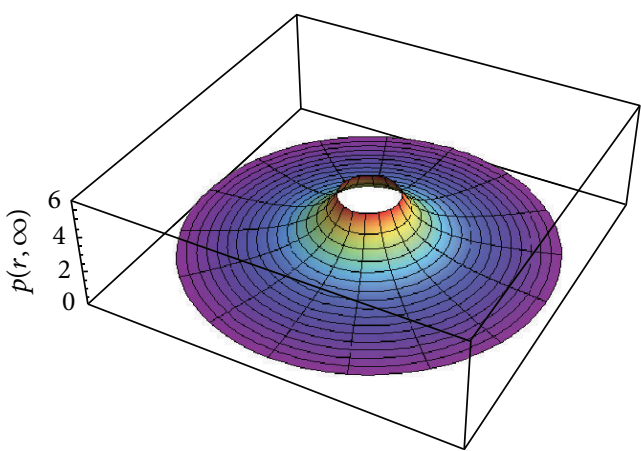

(b) $\rho=0.2$

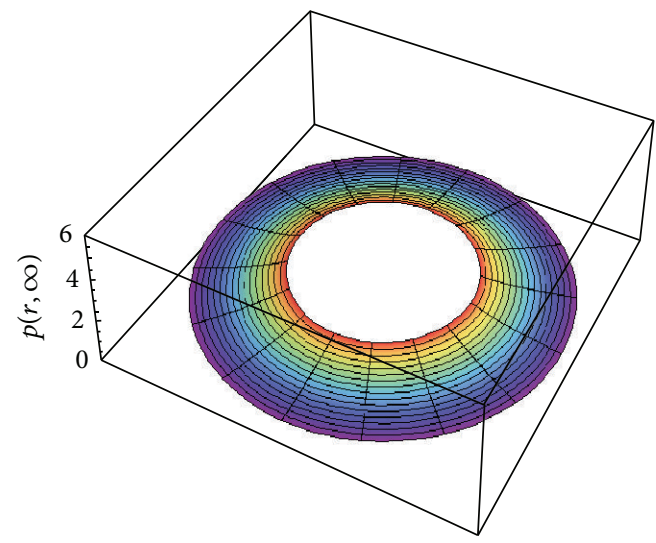

(d) $\rho=1.0$

FIGURE 9: Visualization of contact pressure distributions in the steady state for different parameter $\rho$.

lining process, the pressure values obtained are presented in a standard form (they are referred to the average value obtained for the contact pressure at the initial moment). Moreover, because the working surface of the sensor had the shape of a circle approximately $1 \mathrm{~cm}$ in diameter, it was impossible to measure the contact pressure at the borders of the contact pads (only the neighboring part located approximately $0.5 \mathrm{~cm}$ from them was applied). The obtained experimental values of $E(t)$, friction torque $M_{\mathrm{fr}}(t)$ transmitted by the clutch, and contact pressures $P(R, t)$ were compared with analytical and numerical calculations obtained for the linear wear model.

Experimental studies of wear processes were carried out in a system consisting of two identical friction linings made of a suitable kind of natural cork. The use of this material due to its low abrasion resistance allowed us to carry out the experiment in a relatively short time. Inner radius $R_{1}$ of the contact surface was $11 \mathrm{~mm}$, while the outer radius of the contact surface was $R_{2}=59 \mathrm{~mm}$. Clutch shields with fixed friction linings were pressed with axial force $Q=20 \mathrm{~N}$ and relative sliding angular velocity $\Omega_{r}=70 \mathrm{rad} / \mathrm{s}$. Appropriate measurements were carried out at regular time intervals $\Delta T=$ $60 \mathrm{~s}$ after stopping the system.

Figure 5 shows a decrease of the distance between clutch shields in time obtained experimentally. On the basis of the results obtained it can be assumed that wear of the clutch friction linings is approximately proportional to time $t$ (it is also proportional to the work of the friction force).
Approximating the obtained experimental results it can be assumed that the slope of the experimental relation is equal to the speed of decrease of the distance between clutch shields in the steady state. Then, on the basis of (39) the total wear coefficient can be estimated as follows:

$$
\begin{aligned}
& \frac{d E(\infty)}{d t}=\frac{K^{(w)} Q\left|\Omega_{r}\right|}{2 \pi\left(R_{2}-R_{1}\right)}, \\
& \Longrightarrow K^{(w)}=\frac{2 \pi\left(R_{2}-R_{1}\right)}{Q\left|\Omega_{r}\right|} \frac{d E(\infty)}{d t} .
\end{aligned}
$$

Taking the appropriate values of $Q, \Omega_{r}, R_{1}$, and $R_{2}$, as well as the value of $d E(\infty) / d t=4.9167 \cdot 10^{-7} \mathrm{~m} / \mathrm{s}$ estimated from Figure 5 , in our case the total wear coefficient is equal to $K^{(w)}=1.059 \cdot 10^{-10} \mathrm{~m}^{2} / \mathrm{N}$.

Figure 6 shows the maximum friction torque transmitted by the clutch in a normalized form (the values of relevant friction torques were referred to the values of friction torque for the initial moment). Moreover, the numerically obtained relation describing changes in time of friction torque transmitted by the clutch is shown in accordance with the respective equations provided in Section 3 and adopting the above obtained values which describe the system. Using the dashed line we illustrate also the value calculated from (58), to which decreases the friction torque transmitted by the clutch after settling contact pressure distribution on the surface of the friction linings of the clutch. This 

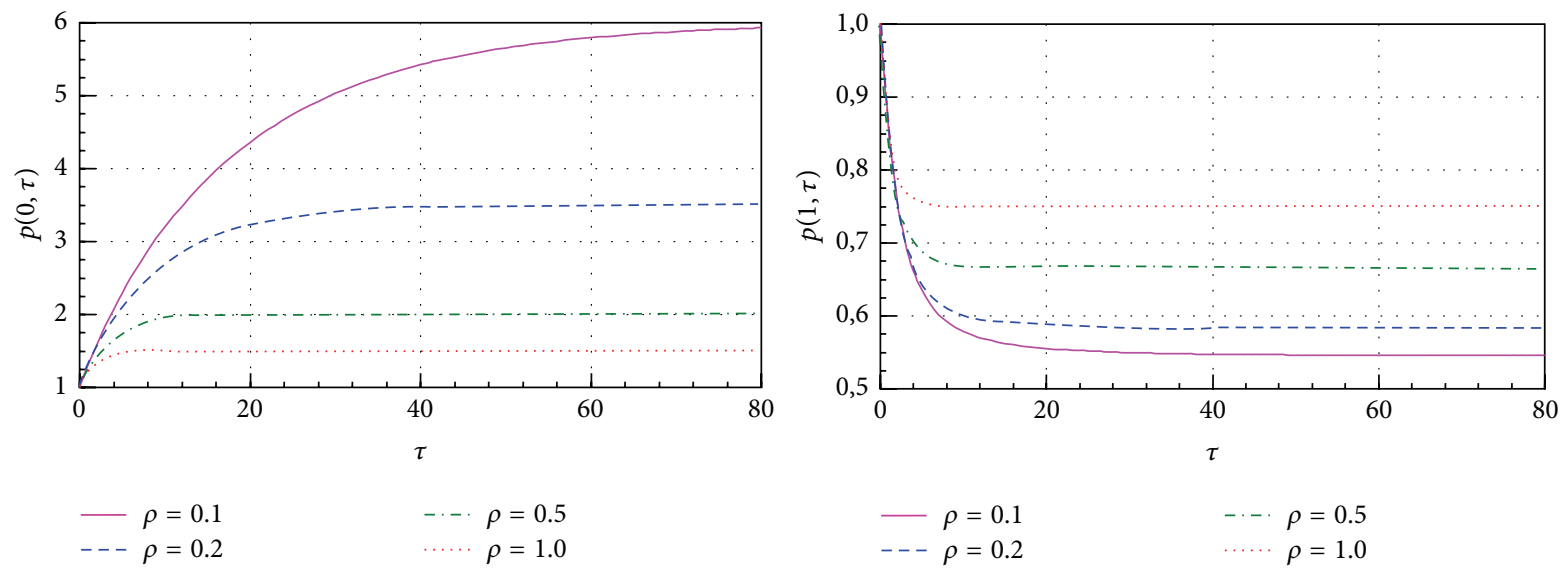

(a) $k^{(w)}=0.5$
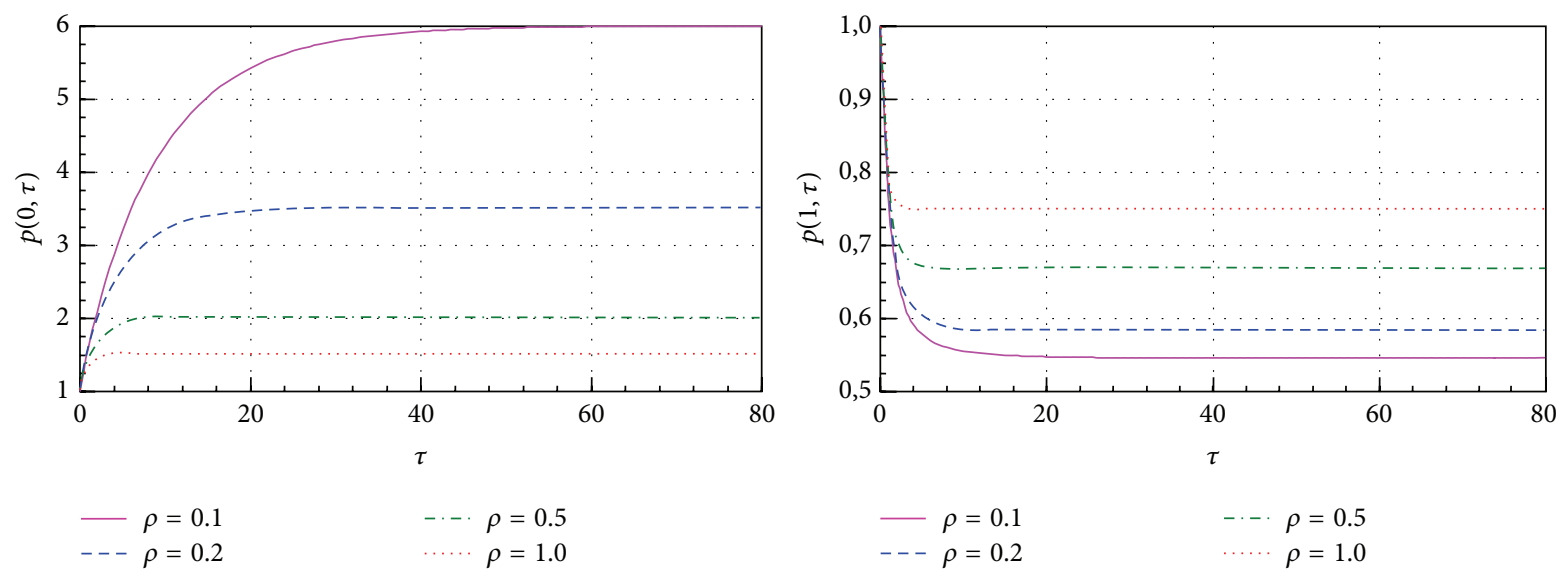

(b) $k^{(w)}=1.0$

FIgURE 10: Changes of contact pressures at the contact surface borders.

does not diminish generality and facilitates comparison with other numerical simulations that will be presented in a similar way. The relationships show that the actual value of torque transmitted by the clutch (in accordance with the numerical simulations) decreases during wear processes of the clutch friction linings. The carried out numerical results allow estimating the steady state for this system. It is equal approximately to $30 \mathrm{~min}$ of wear duration. Experimental results show, however, that the steady state had been achieved previously. It is also seen that the experimental results do not agree precisely with the numerical solution. However, similarly to the numerically obtained simulation, the results show a characteristic decrease of the real value of friction torque transmitted by the friction clutch.

Figure 7 shows contact pressures on the contact surface of friction clutch linings along radius $R$. The experimental results are presented only for the initial time and steady state, whereby it is assumed that the steady state occurred after time equal to $30 \mathrm{~min}$ from starting of the wear process. Although the presented experimental results do not agree with a relatively high accuracy with the results obtained analytically, it may be important to note qualitative changes of the contact pressure distribution in accordance with the analytical predictions. Namely, at the initial moment of time the contact pressure distribution is approximately uniform over the entire contact surface. Moreover, during wear processes of the friction linings, the contact pressure distribution changes, but it is important that at the inner border of the contact surface pressures increase, while at the outer contact surface they decrease.

5.4. Summary of Experimental Results. The presented experimental investigations confirm and validate the proposed mathematical models describing wear processes that occur in a mechanical friction clutch. The mathematical models describing wear processes of friction linings were verified experimentally, although the results were compared only with the analytical and numerical calculations obtained for the linear wear model. However, it was shown that reduction of the friction torque transmitted by the clutch for a constant force pressing the shields or changing the contact pressure distributions agreed with the proposed mathematical model. The relatively simple experiments revealed that the proposed mathematical models are good enough to describe the phenomena occurring in the real objects. Therefore, the proposed models can be used for numerical simulations in a 

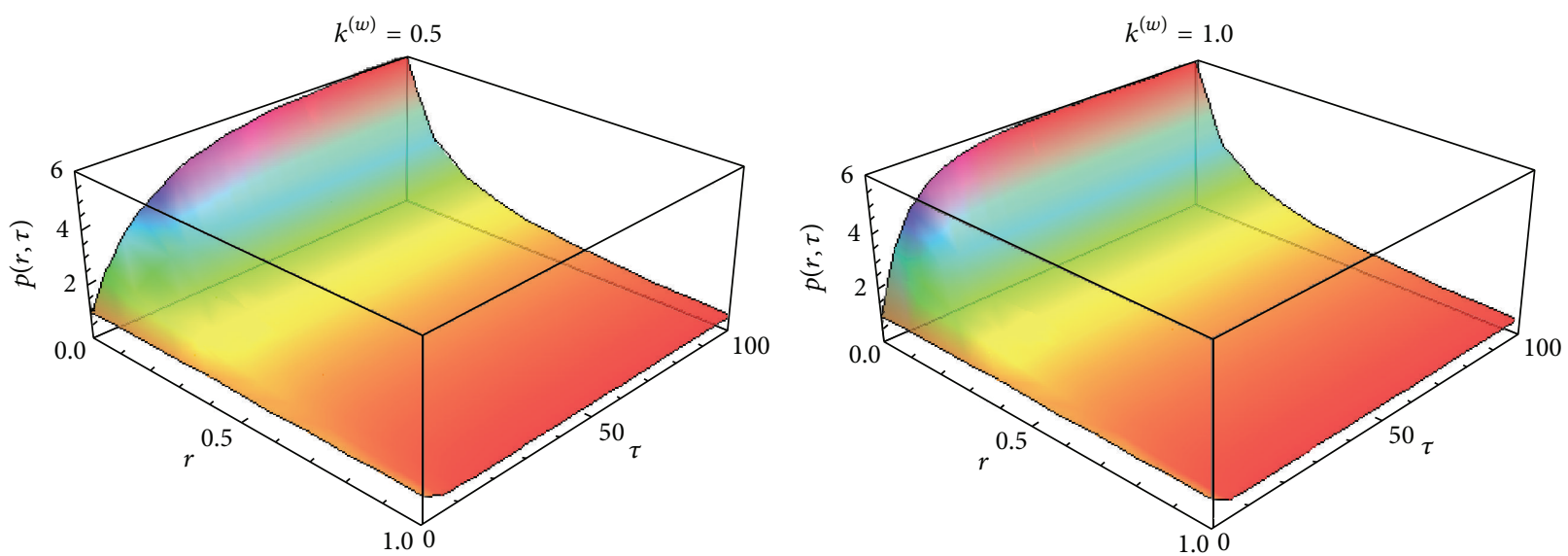

(a) $\rho=0.1$
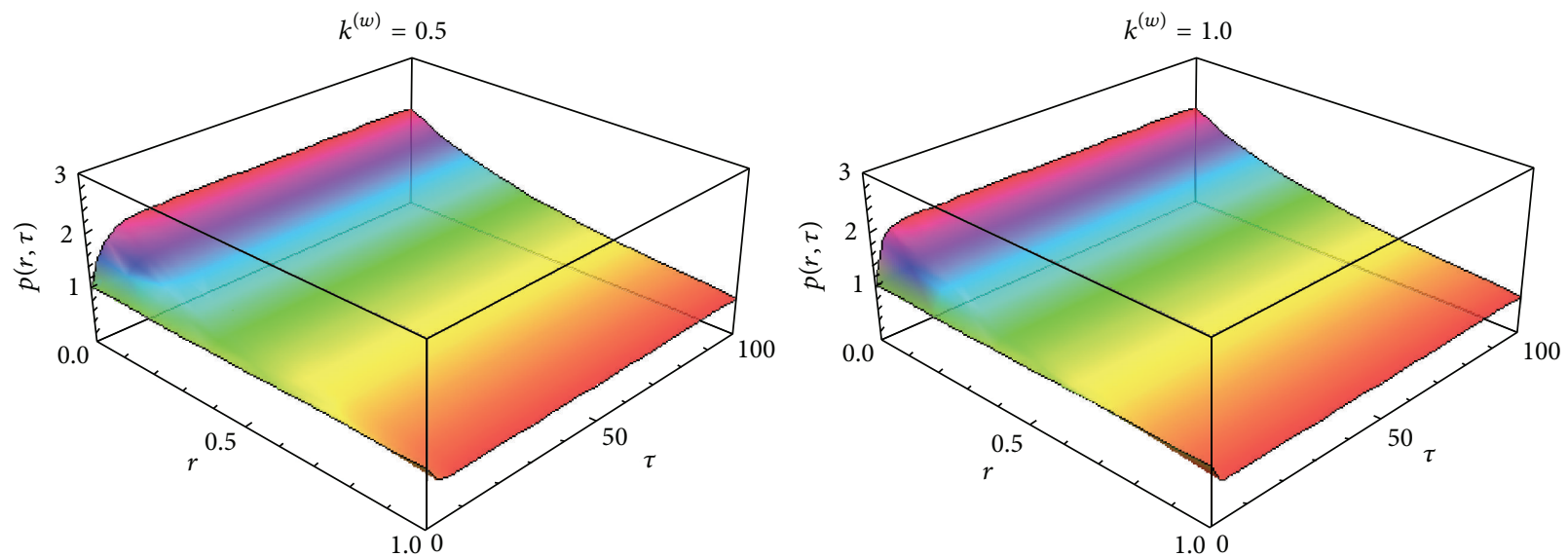

(b) $\rho=0.5$

FIgURE 11: Time evolutions of the contact pressure distributions.

wider range of parameters which are presented in the next section. These two applied types of modern experiments (the experiment and computer simulation) complement each other. The experiment allows us to verify the proposed mathematical models, whereas computer simulations enable researches in a wider range of parameters describing the actual object of study.

\section{Numerical Results}

In this section a numerical analysis of the proposed mathematical models is carried out in a wider range of parameters. The analysis of wear processes of the friction clutch linings using both differential and integral wear model in this clutch is given. We introduced the following assumptions: $k_{1}^{(w)}(T(r, \tau))=k_{1}^{(w)}=$ constant, $k_{2}^{(w)}(T(r, \tau))=k_{2}^{(w)}=$ constant, and $f(\omega(\tau), r, T(r, \tau))=f=$ constant (see also our previous works $[33,34])$.

6.1. Differential Wear Model. First we analyze wear processes of friction clutch linings using the differential wear model. We focus on a symmetrical system consisting of two identical clutch friction linings, and therefore in the numerical simulations we take the following assumptions: $\alpha_{1}=\alpha_{2}=$ $\alpha, \beta_{1}=\beta_{2}=\beta, k_{1}^{(w)}=k_{2}^{(w)}$, and $k_{1}^{(w)}+k_{2}^{(w)}=k^{(w)}$. The obtained results are also valid for the case when one of the clutch shields is not covered by the friction lining, while the other one is covered by a lining with wear coefficient equal to $k^{(w)}$. For $\alpha=1$ and $\beta=1$ we have linear Archard's wear model [5]. For further numerical calculations we take $k^{(w)}=0.5$.

Figure 8 presents contact pressure distributions in the steady state for various geometric parameters $\rho$ obtained analytically on the basis of (54). Moreover, the contact pressure distribution in initial time $(p(r, 0)=1)$ is plotted in Figure 8. The reported relation shows that these distributions depend on parameter $\rho$ which determines the considered system geometry. At the initial time instant contact pressure distributions are uniform over the entire contact surface, while they change during time evolution. In the steady state, contact pressure takes the specific distributions versus parameter $\rho$. For large values of $\rho$ the contact pressure distribution does not change significantly in comparison to the initial distribution, whereas the differences are significant for smaller values of this parameter.

Contact pressure distributions determined analytically in (54) are also shown in Figure 9 as visualizations in polar 


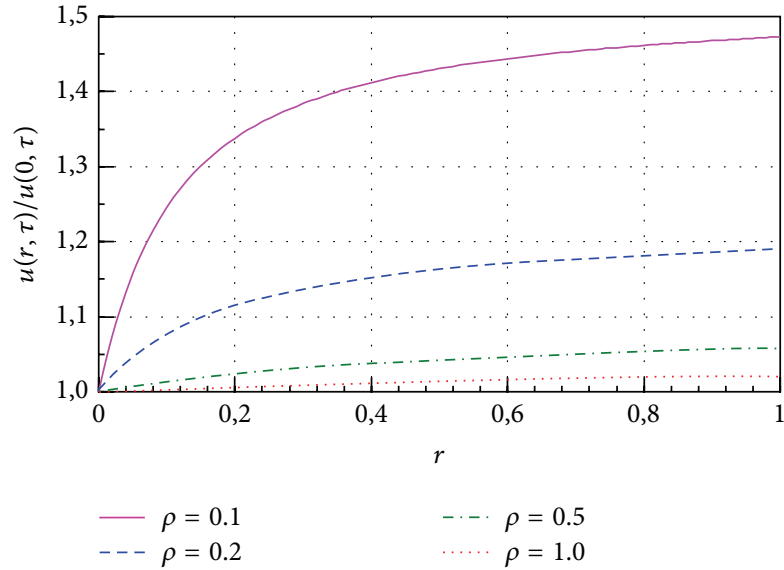

(a) $\tau=50$

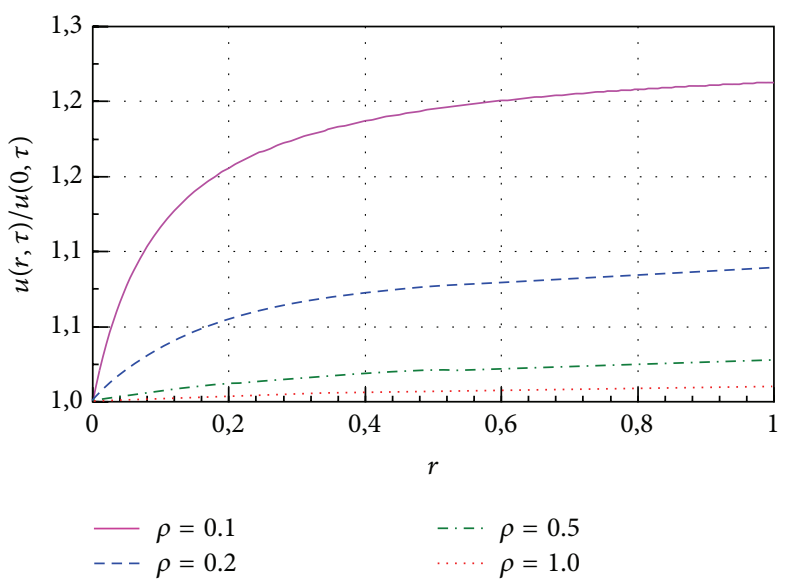

(b) $\tau=100$

FIGURE 12: Wear distributions in transient states.

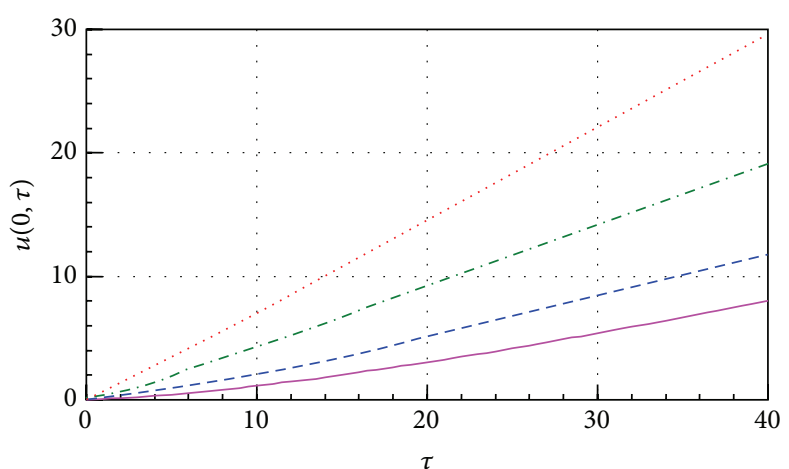

$-\rho=0.1$

$--\rho=0.2$

$-\cdot \rho=0.5$

$\rho=1.0$

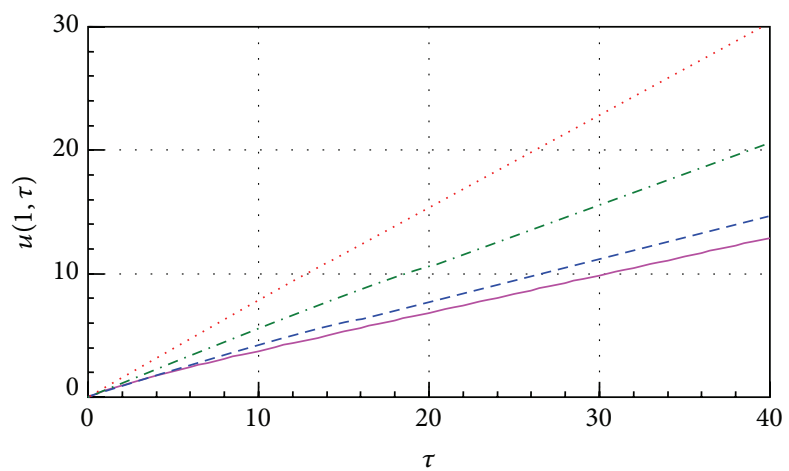

$\begin{aligned}-\rho & =0.1 \\ --\rho & =0.2\end{aligned}$

-.-. $\rho=0.5$

$\rho=1.0$

(a) $k^{(w)}=0.5$
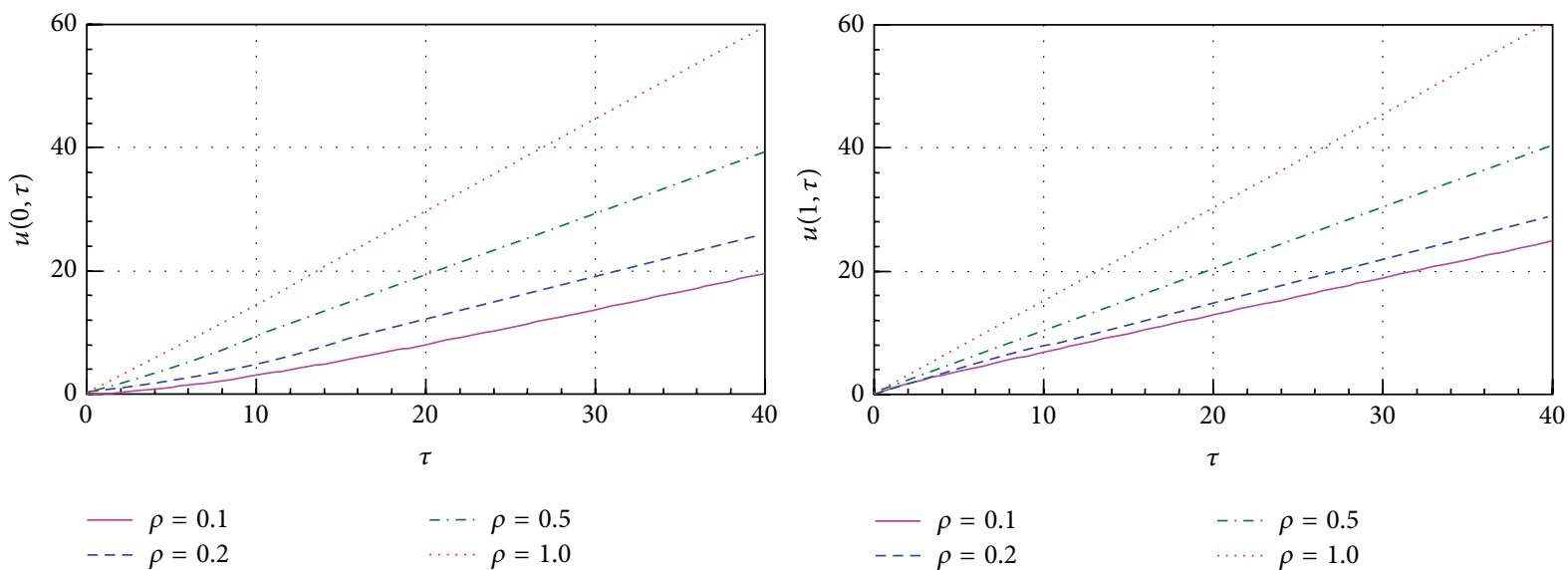

(b) $k^{(w)}=1.0$

FIGURE 13: Time evolutions of total wear at the contact surface borders.

coordinates, where the domain is the surface corresponding to the contact surfaces of friction linings for appropriate geometric parameter $\rho$.

Contact pressures at the contact surface borders $(r=$ 0 and $r=1$ ) for different $\rho$ are shown in Figure 10. For comparison also the results obtained for wear coefficient $k^{(w)}=1.0$ are presented. During wear processes, with an increase of time, contact pressures $p(0, \tau)$ increase to reach finally a steady state value depending on $\rho$. Contact pressures $p(1, \tau)$ decrease also reaching a steady state value which 

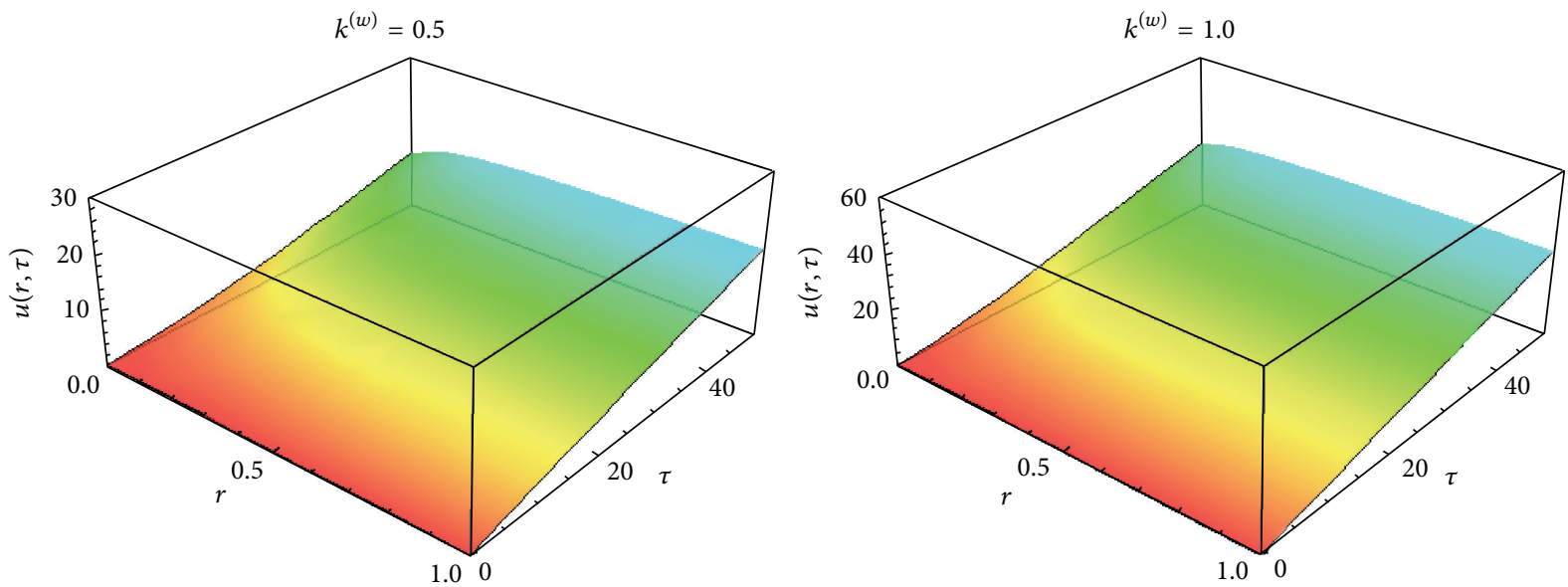

(a) $\rho=0.1$
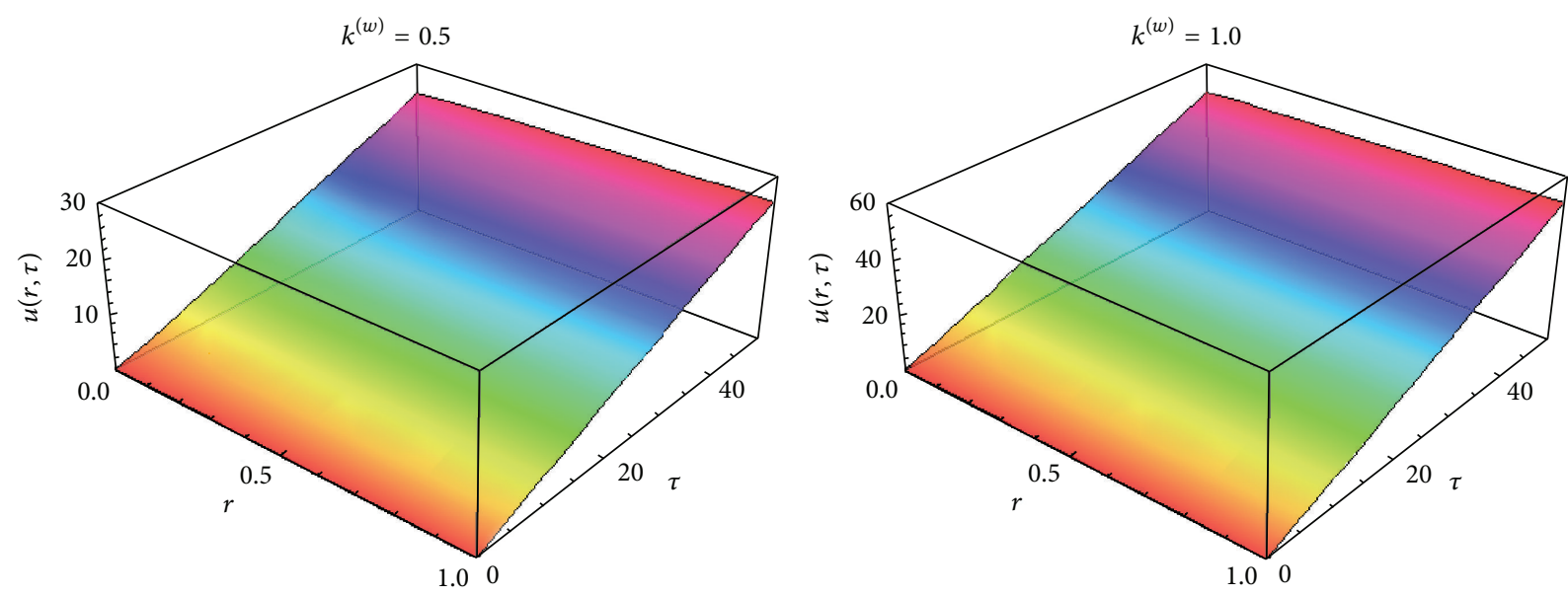

(b) $\rho=0.5$

FIGURE 14: Time evolutions of total wear distributions of friction linings.

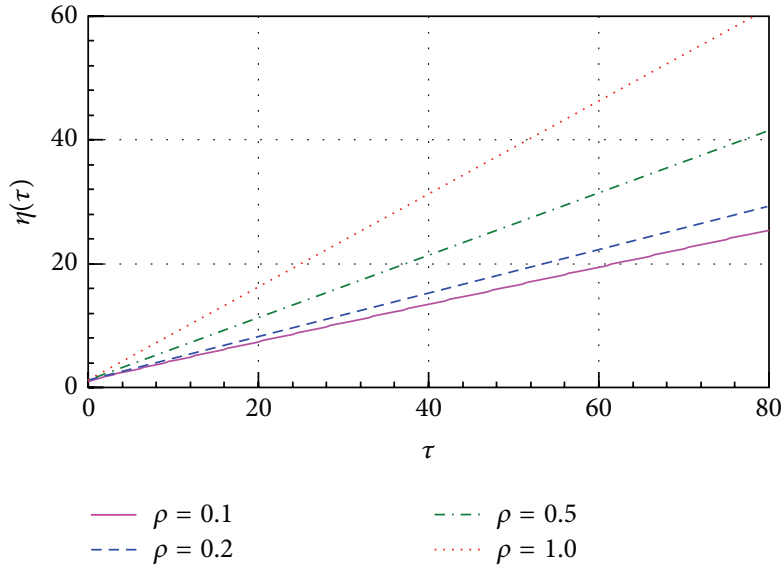

(a) $k^{(w)}=0.5$

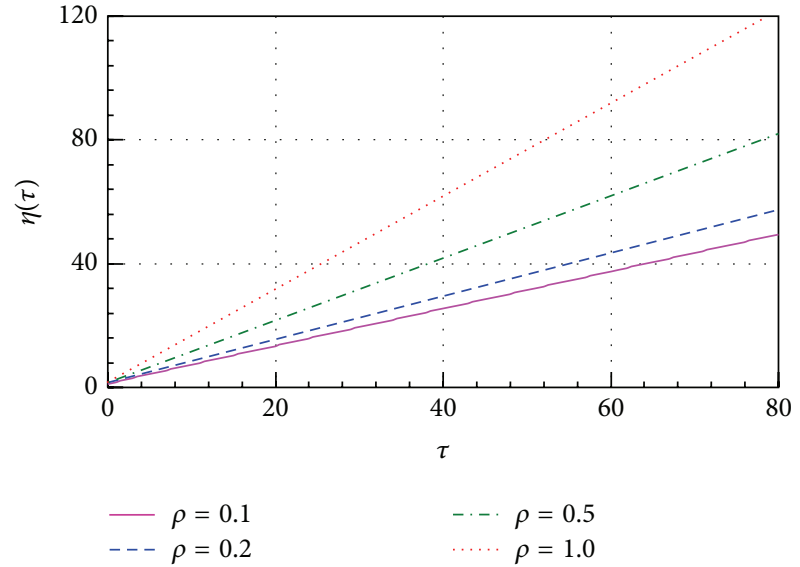

(b) $k^{(w)}=1.0$

FIGURE 15: Time evolutions of decrease of the distance between clutch shields. 


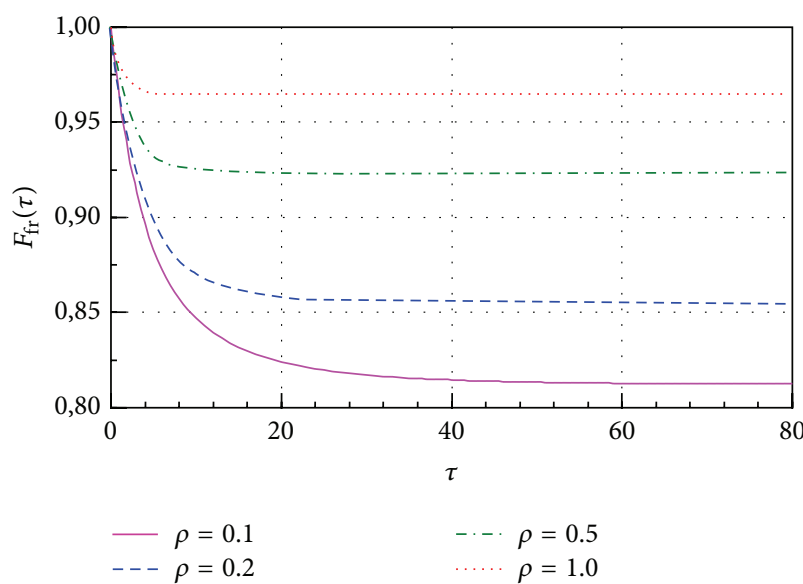

(a) $k^{(w)}=0.5$

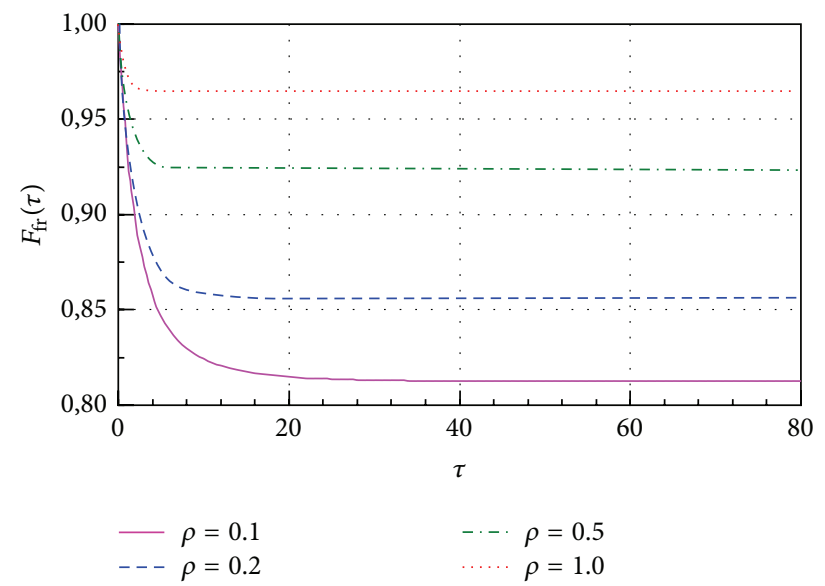

(b) $k^{(w)}=1.0$

FIGURE 16: Changes of friction torque transmitted by the clutch.

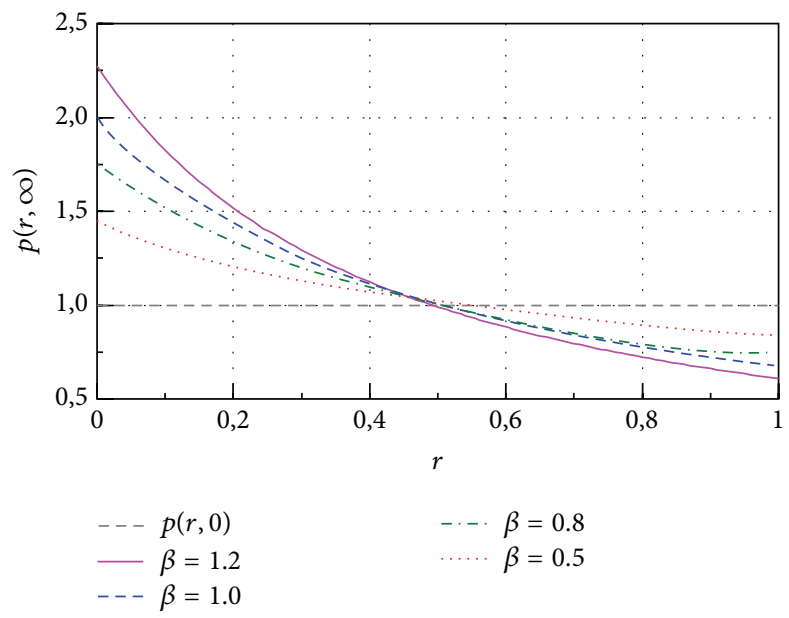

FIGURE 17: Contact pressure distributions in the steady state for various $\beta$.
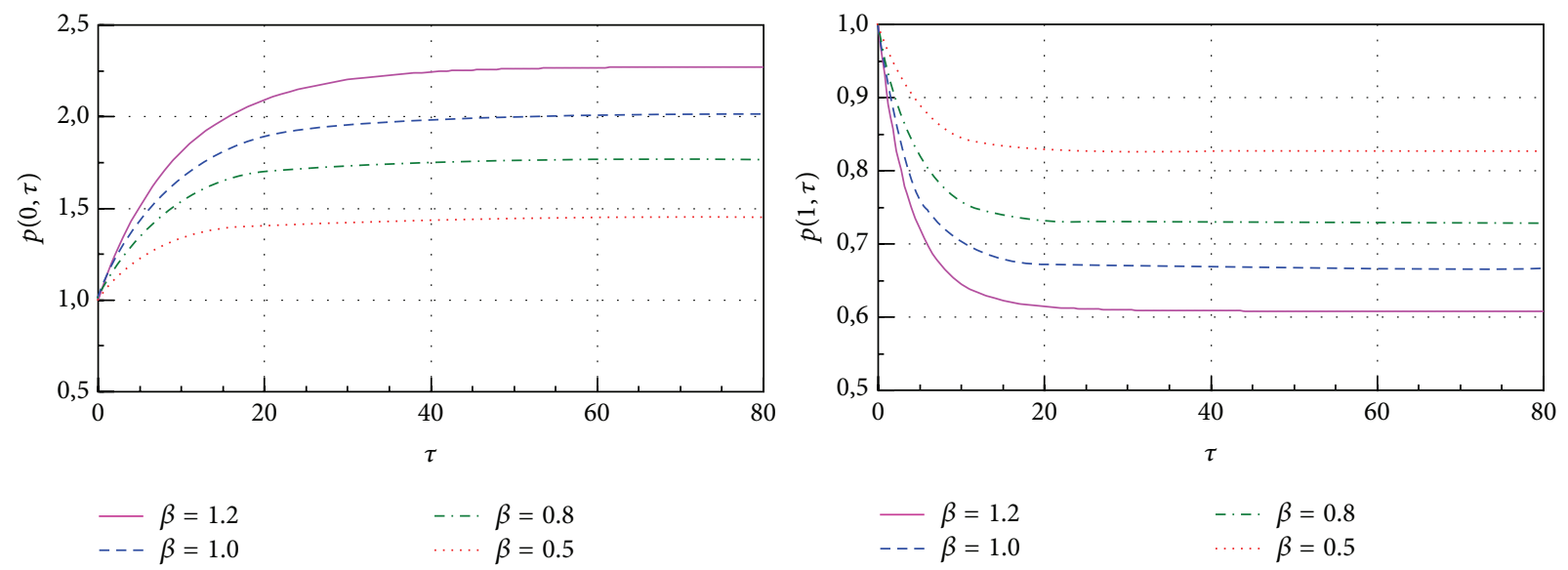

$-\beta=1.2$

-.. $\beta=0.8$

$\beta=0.5$

$\begin{aligned}-\beta & =1.2 \\ --\beta & =1.0\end{aligned}$

$\beta=0.8$
$\beta=0.5$

FIGURE 18: Time evolutions of contact pressures at the contact surface borders ( $r=0$ and $r=1)$. 


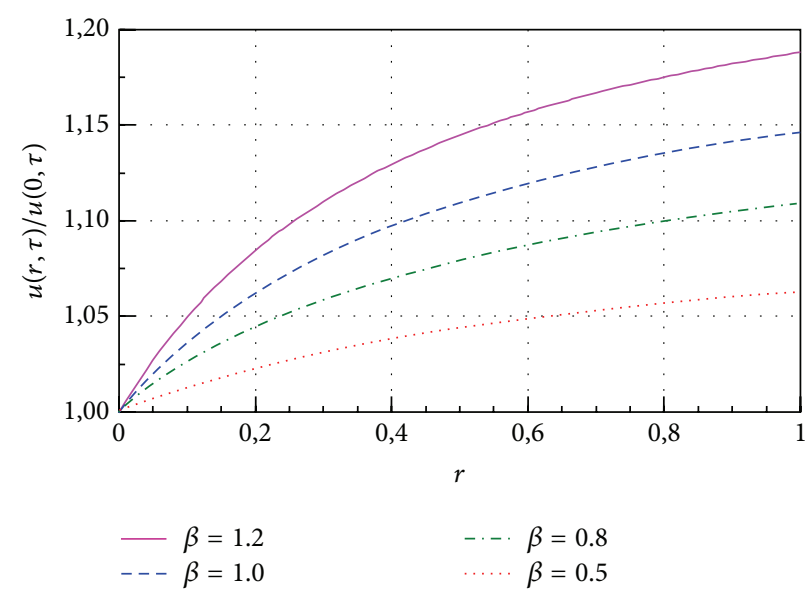

(a) $\tau=50$

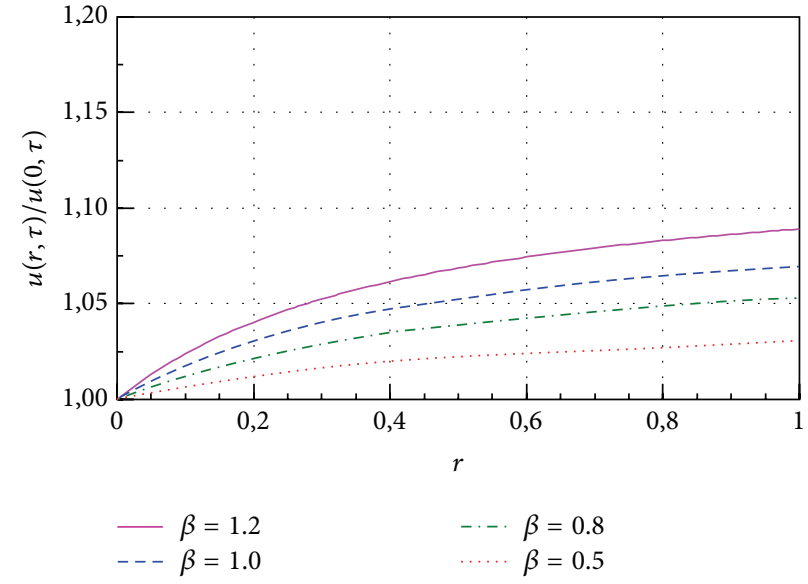

(b) $\tau=100$

FIGURE 19: Total wear distributions in transient states for various times $\tau$.
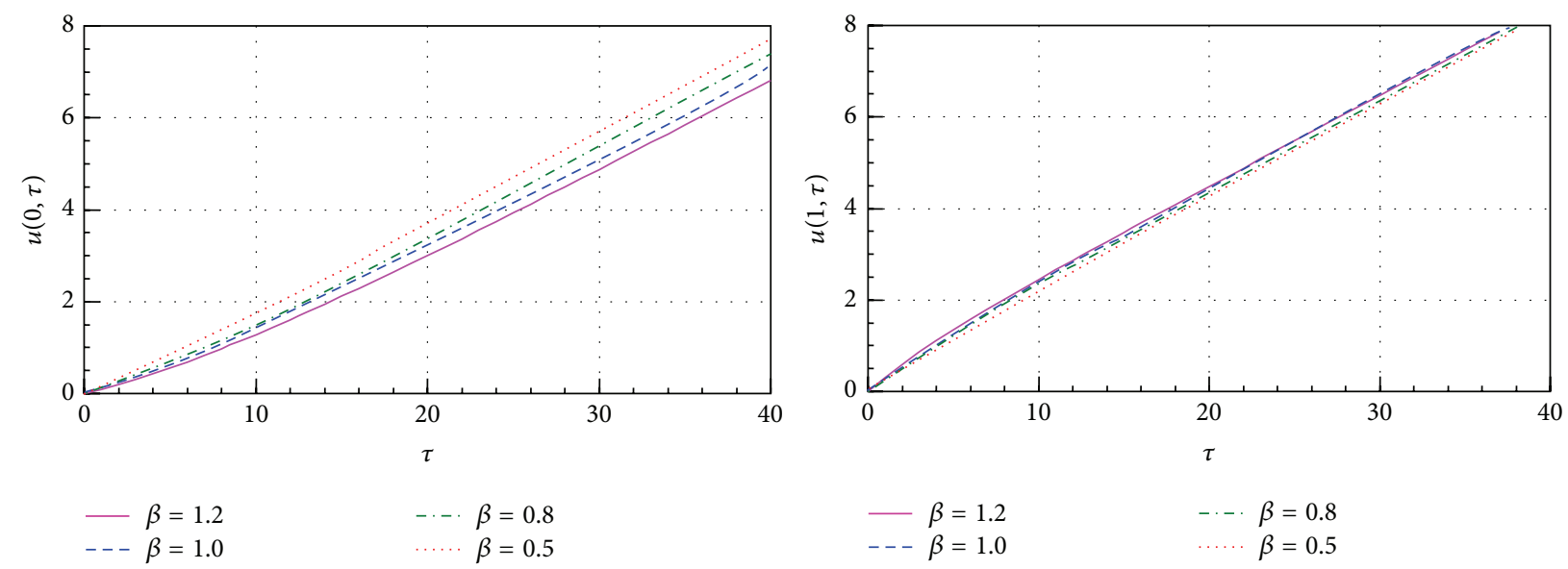

FIgURE 20: Time evolutions of total wear at the contact surface borders $(r=0$ and $r=1)$.

depends on $\rho$. Moreover, the steady state is reached faster for larger values of the wear coefficient $k^{(w)}$.

Time evolutions of the contact pressure distributions as a function of radius $r$ and time $\tau$ are shown in Figure 11. Also here the results obtained for wear coefficient $k^{(w)}=1.0$ are presented. For all presented cases the contact pressure distributions at the initial time instant are uniform over the entire surface of contact and they change during wear processes. They reach the steady state depending on parameter $\rho$. The speed of setting of these distributions is larger for higher values of wear coefficient $k^{(w)}$.

Figure 12 shows the distributions of total wear of friction linings in transient states for different values of the nondimensional time $\tau$. It can be seen that the wear distributions are different for different values of geometric parameter $\rho$. These distributions also change over time. Furthermore, it is worth noting that in any case (irrespective of $\rho$ and $\tau$ ) wear of the friction lining is always the largest at the outer border of the contact surface (for $r=1$ ). Figure 13 presents changes of the total wear at the contact surface borders $(r=0$ and $r=1)$.
For comparison, also the results obtained for wear coefficient $k^{(w)}=1.0$ are presented.

Time evolutions of total wear distributions of friction linings are shown in Figure 14. At the initial time wears at each point of the contact surface are zero which means that the wears increase. The speed of wear $d u(0, \tau) / d \tau$ at the initial time instant is slightly less than the value determined later. In turn, the speed of wear $d u(1, \tau) / d \tau$ at the initial time instant is slightly greater than the value determined later. On the other hand, the speed of wear is larger for larger values of $\rho$ and $k^{(w)}$.

Time evolutions governing the decrease of the distance between clutch shields (for various values of coefficient $k^{(w)}$ ) are shown in Figure 15. The decrease of the distance between clutch shields increases faster for larger values of the geometry parameter $\rho$ and for larger values of parameter $k^{(w)}$. Furthermore, in the steady state the mentioned process increases linearly, like in the case of total wear.

Changes in the friction torque transmitted by the clutch are presented in Figure 16 for $k^{(w)}=0.5$ and $k^{(w)}=1.0$. One may see that during wear processes the friction torque 


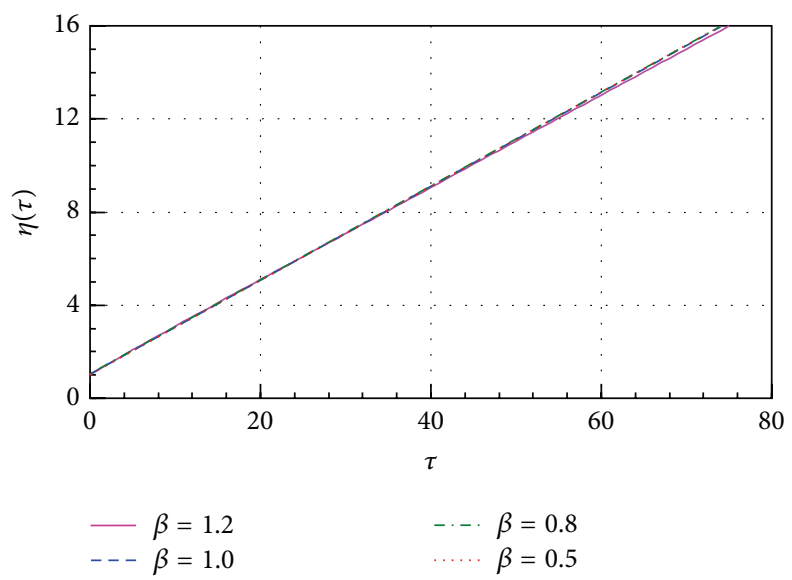

FIgURE 21: Decrease of the distance between clutch shields for various $\beta$.

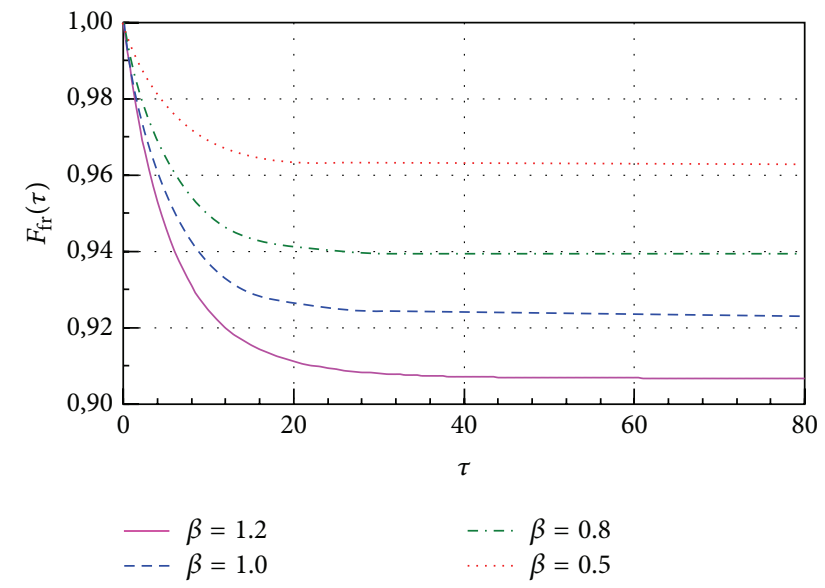

FIGURE 22: Changes of friction torque transmitted by the clutch for various $\beta$.

transmitted by the clutch decreases as a result of changes in the contact pressure distributions. For a fixed contact pressure distribution and in the steady state the friction torque transmitted by the clutch reaches a constant value. For smaller values of geometric parameter $\rho$ a relative change of the friction torque is larger.

In this paper the case of nonlinear differential wear model is also considered. In further calculations we take $\rho=0.5$ and $k^{(w)}=0.2$. First, we consider the case of $\alpha=1$ and $\beta \neq 1$. Contact pressure distributions, wear distributions, decrease of the distance between clutch shields, and the friction torque transmitted by the clutch for various values of $\beta$ parameter are presented: contact pressure distributions in the steady state (Figure 17), time evolutions of contact pressures at the contact surface borders $r=0$ and $r=1$ (Figure 18), total wear distributions in transient states for various times $\tau$ (Figure 19), time evolutions of total wear at the contact surface borders $r=0$ and $r=1$ (Figure 20), decrease of the distance between clutch shields (Figure 21), and changes of the friction torque transmitted by the clutch (Figure 22).

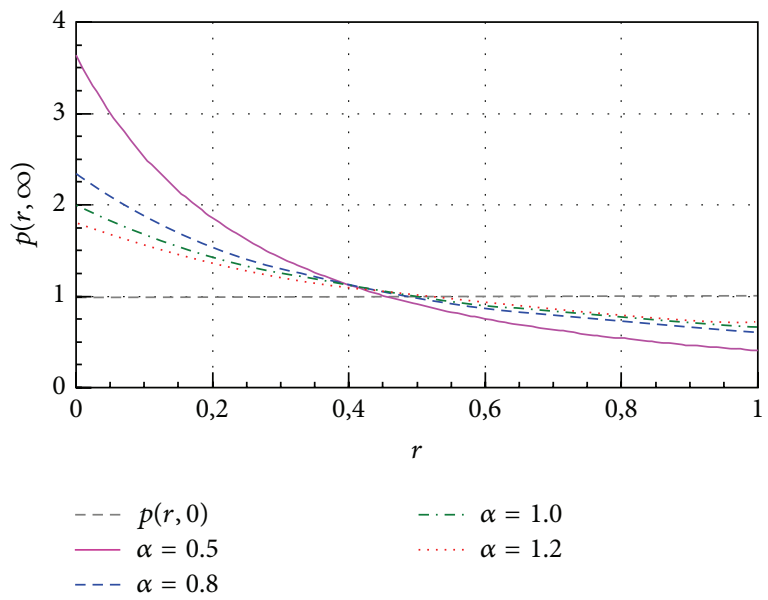

FIGURE 23: Contact pressure distributions (steady state) for different values of $\alpha$.

For the differential wear model appropriate results illustrating contact pressures, wears, decrease of the distance between clutch shields, and friction torque transmitted by the clutch are also presented for different values of parameter $\alpha$ : contact pressures in the steady state (Figure 23), time evolutions of contact pressures at the contact surface borders $r=0$ and $r=1$ (Figure 24), total wear distributions in transient states (Figure 25), time evolutions of wear at the contact surface borders $r=0$ and $r=1$ (Figure 26), decrease of the distance between clutch shields (Figure 27), and changes of friction torque transmitted by the clutch (Figure 28).

The presented model allows us to study wear processes of clutch friction linings for arbitrary values of parameters $\alpha$ and $\beta$. In addition, the proposed model can also be used to analyze these processes in a nonsymmetric case, namely, for $k_{1}^{(w)} \neq k_{2}^{(w)}, \alpha_{1} \neq \alpha_{2}$, and $\beta_{1} \neq \beta_{2}$.

6.2. Integral Wear Model. Below, we consider a numerical analysis of wear processes of the friction clutch linings 

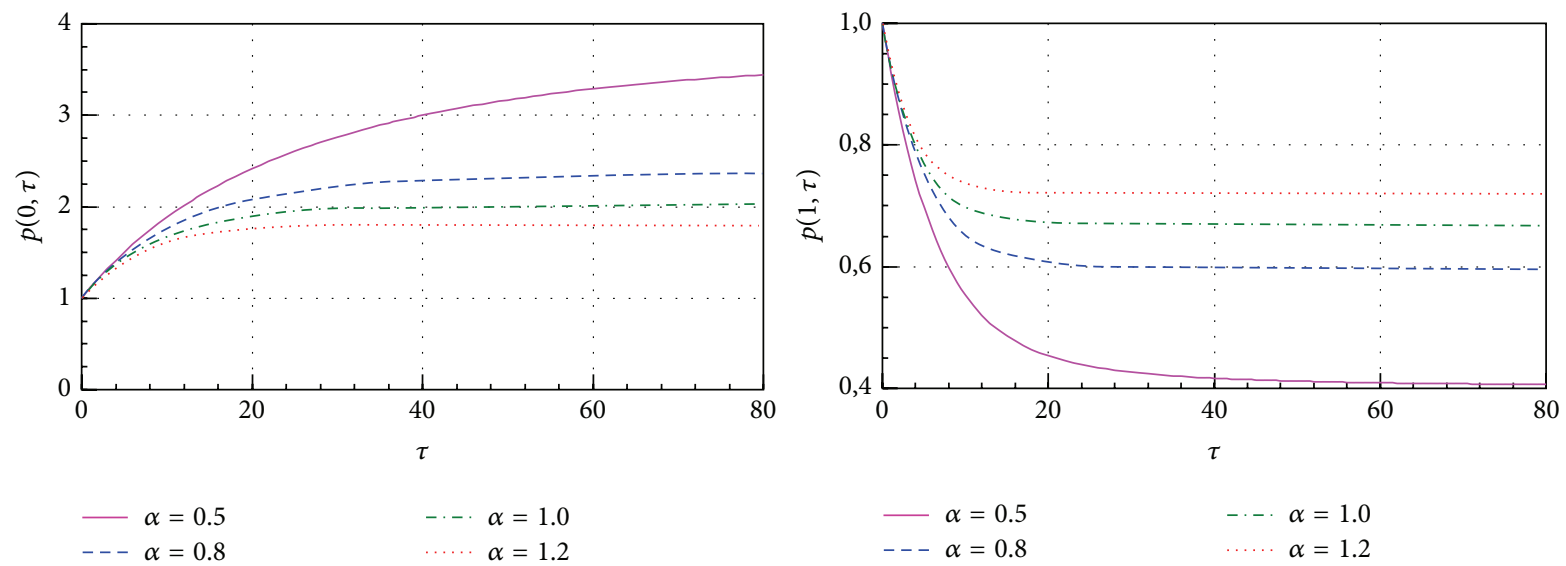

FIGURE 24: Time evolutions of contact pressures at the contact surface borders $(r=0$ and $r=1)$.

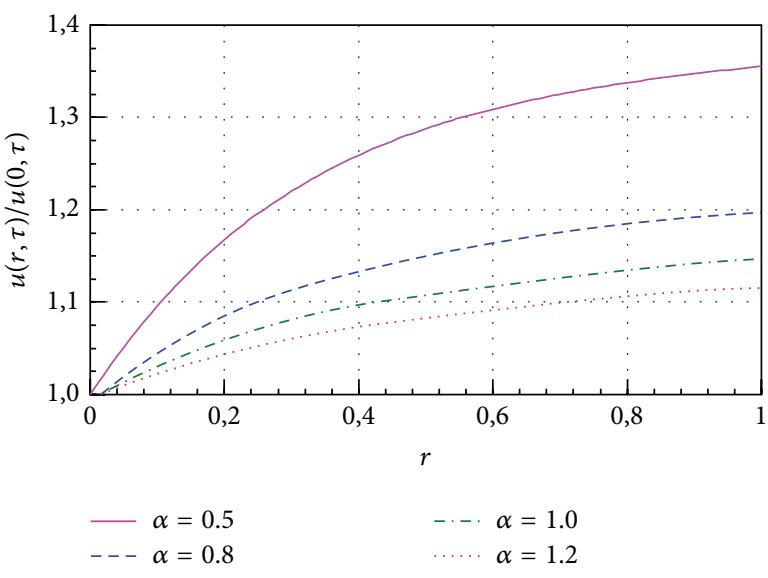

(a) $\tau=50$

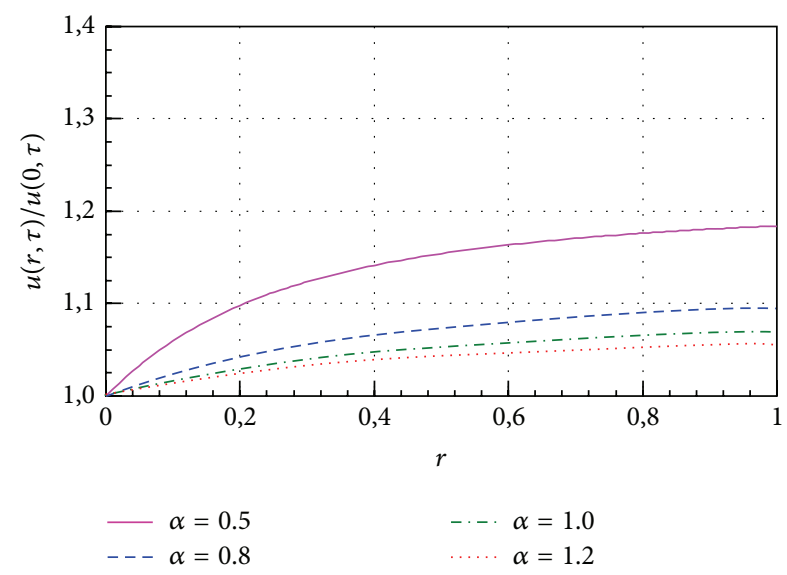

(b) $\tau=100$

FIGURE 25: Total wear distributions in transient states.

using the integral wear model. We focus on a symmetrical system consisting of two identical clutch friction linings. For this reason, in numerical simulations we take the following assumptions: $K_{1}(\tau, \xi)=K_{2}(\tau, \xi)=K(\tau, \xi), k_{1}^{(w)}=k_{2}^{(w)}$, and $k_{1}^{(w)}+k_{2}^{(w)}=k^{(w)}$. The obtained results are also valid for the case when one of the clutch shields is not covered with the friction lining, while the other one is covered with a lining with wear coefficient equal to $k^{(w)}$. For $K(\tau, \xi)=1$ we have a linear wear model (according to Archard [5]) written in the integral form. The obtained results are identical to those obtained earlier in the case of the differential wear model for $\alpha_{1}=\alpha_{2}=1$ and $\beta_{1}=\beta_{2}=1$. For this reason below we present results obtained for the nonlinear integral wear model, and for further numerical calculations we take $K(\tau, \xi)=\exp [-\gamma(\tau-\xi)], \rho=0.5$, and $k^{(w)}=0.2$.

Figure 29 shows contact pressure distributions in the steady state for various values of parameter $\gamma$. The contact pressure distributions are also shown in Figure 30 in polar coordinates. Observe that these distributions depend on parameter $\gamma$. At the initial time instant the contact pressure distribution is uniform over the entire contact surface, while with time it changes. Contact pressure takes the specific distributions in the steady state depending on parameter $\gamma$. It does not change significantly for large values of parameter $\gamma$ in comparison to the initial distribution, while differences are significant for smaller values of this parameter.

Changes of contact pressure distributions for various values of parameter $\gamma$ are shown in Figure 31, whereas changes of the total wear distributions for various values of parameter $\gamma$ are shown in Figure 32. For all of the presented cases, the contact pressure distributions at the initial time instant are uniform over the entire surface of contact and wears are equal to zero. During time and wear processes both contact pressure distributions and wear distributions change depending on parameter $\gamma$. For larger values of parameter $\gamma$ the wears are smaller.

Figures 33 and 34 show contact pressures $p(0, \tau), p(1, \tau)$ and wears $u(0, \tau), u(1, \tau)$ on the contact surface borders ( $r=0$ and $r=1$ ) as functions of nondimensional time $\tau$. With time (during wear processes) contact pressure $p(0, \tau)$ increases reaching a steady state value depending on parameter $\gamma$. In turn, contact pressure $p(1, \tau)$ decreases reaching also a steady state value that depends on parameter 

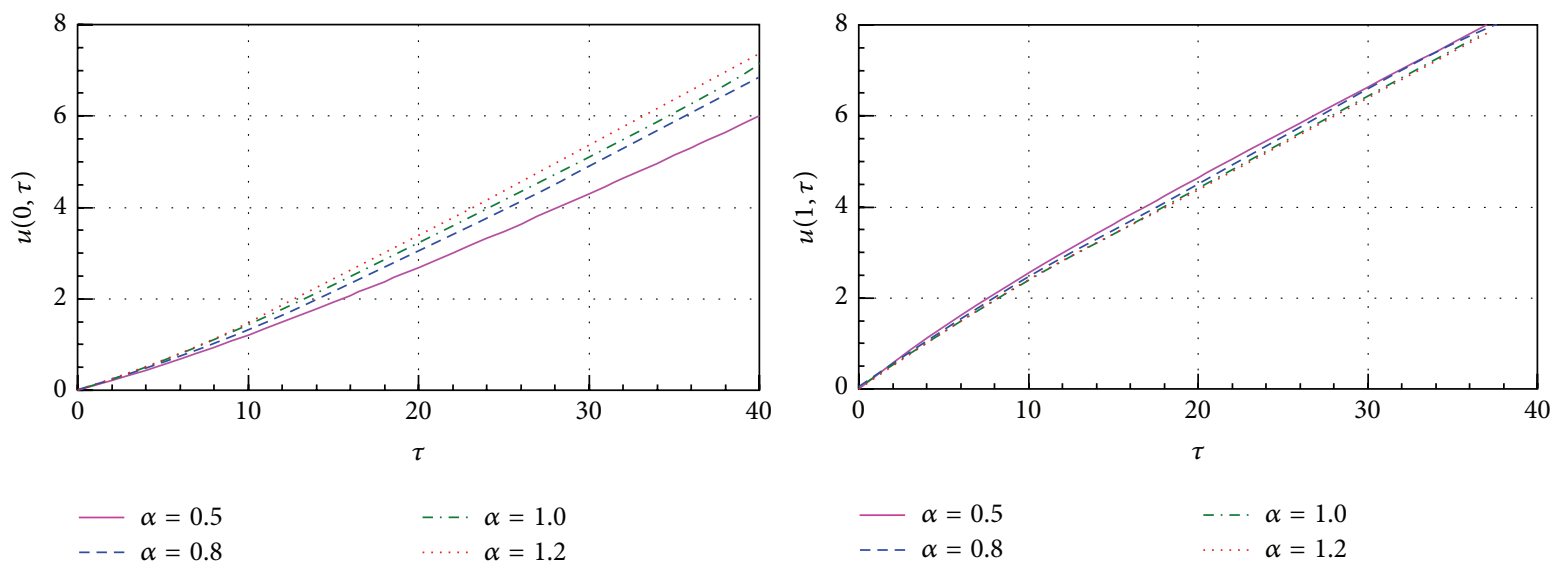

FIgURE 26: Time evolutions of wear at the contact surface borders.

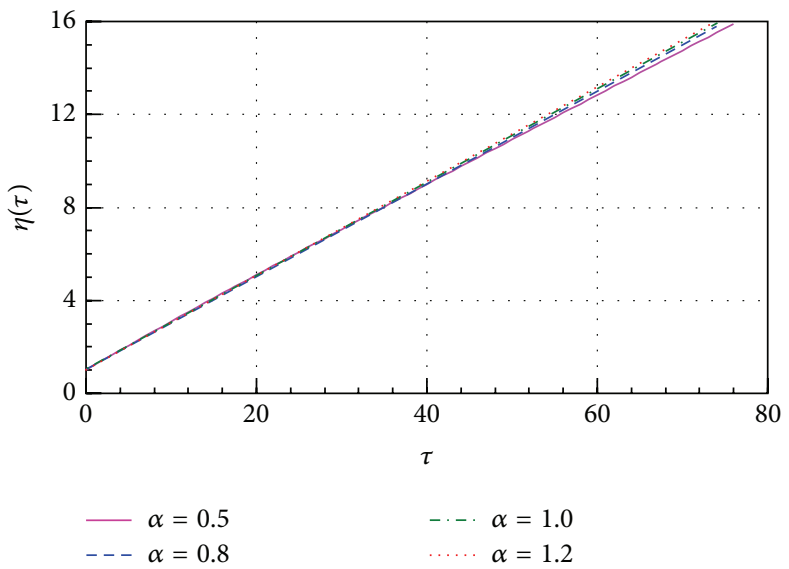

FIGURE 27: Decrease of the distance between clutch shields for various $\alpha$.

$\gamma$. For $\gamma=0$ in the steady state, the wears linearly increase (this is a linear wear model according to Archard [5]). In turn, for $\gamma>0$ in time the speeds of wear decrease asymptotically reaching a constant value which depends on parameter $\gamma$.

Figures 35 and 36 show, respectively, a decrease of the distance between clutch shields and changes of friction torque transmitted by the clutch for various values of parameter $\gamma$ as a function of $\tau$. During wear processes the distance between clutch shields and friction torque transmitted by the clutch decreases. For $\gamma=0$ (linear wear model) the distance between clutch shields increases linearly, whereas for $\gamma>0$ the speed of distance decrease between clutch shields approaches zero. As a result of changes in contact pressure distributions also the friction torque transmitted by the clutch decreases. In the steady state friction torque transmitted by the clutch achieves a constant value depending on $\gamma$.

The presented model allows us to study wear processes of clutch friction linings for arbitrary values of parameter $\gamma$. In addition, the proposed model also enables analysis of these processes in the case of a nonsymmetric system, namely, in general for $k_{1}^{(w)} \neq k_{2}^{(w)}$ and $K_{1}(\tau, \xi) \neq K_{2}(\tau, \xi)$.

\section{Summary and Final Conclusions}

The paper is devoted to the mathematical modeling, theoretical and numerical analysis, and experimental verification of wear processes occurring on the contact surface of friction linings in a mechanical friction clutch. A detailed mathematical description, theoretical analysis, nondimensional form, numerical algorithms and simulations, and experimental verifications are shown.

The proposed models describing wear processes take into account a general nonlinear model of wear, where the wear is modeled via a nonlinear- (power-) type function of contact pressure and relative sliding velocity with rates depending on the wear model and the step of lubricating and spreading on contacting surfaces. Besides, the wear model in the integral form which takes into account a gradual decrease of the speed of wear of friction linings is developed and applied.

The obtained experimental data confirm and validate the presented mathematical models of wear processes in the considered system. Although the proposed models were verified and compared only with calculations obtained for the linear wear model and experimental results do not agree 


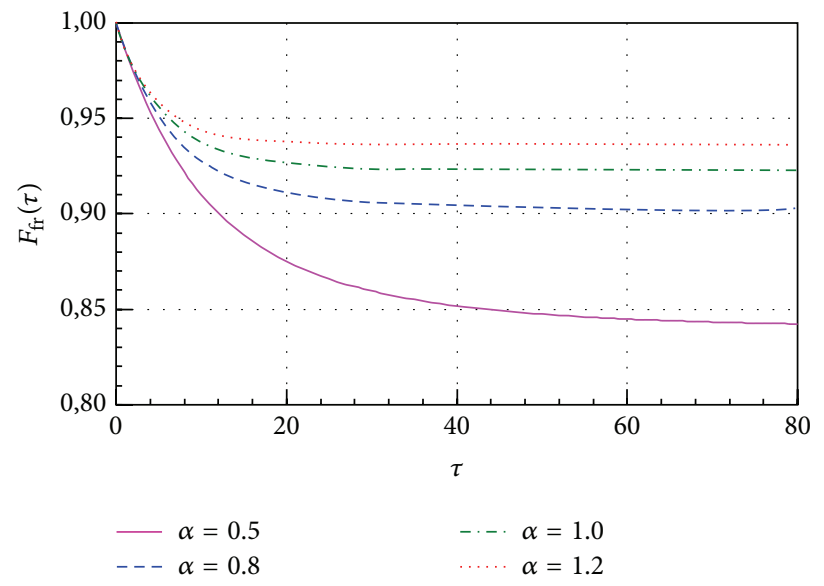

Figure 28: Changes of friction torque transmitted by the clutch for various $\alpha$.

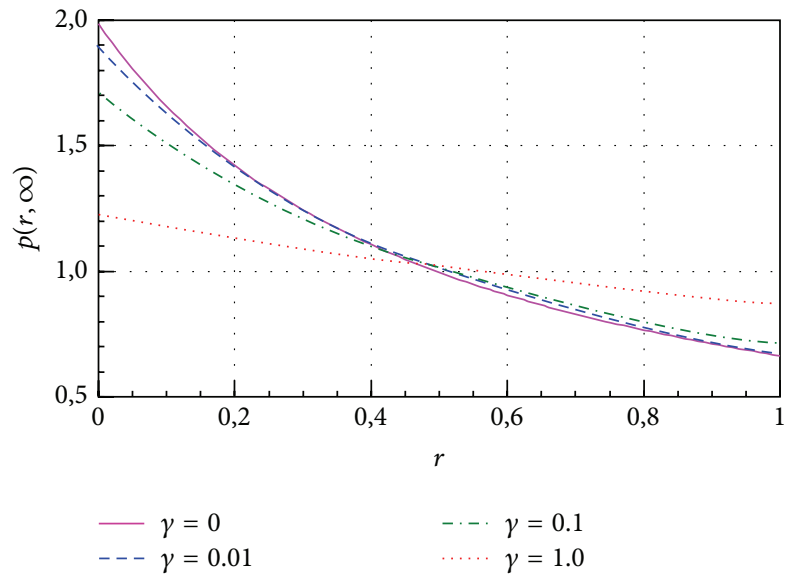

FIGURE 29: Contact pressure distributions in the steady state for various values of $\gamma$.

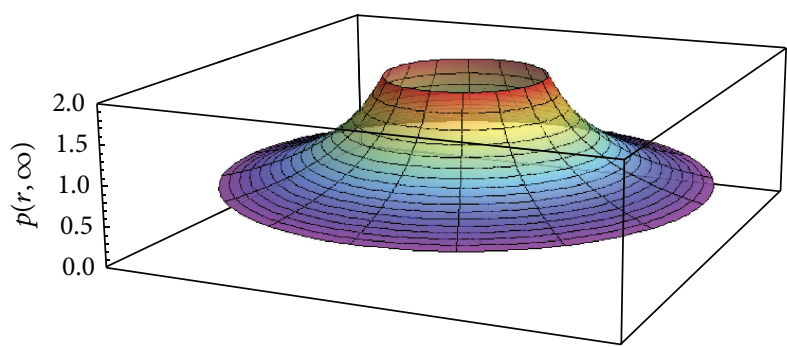

(a) $\gamma=0$

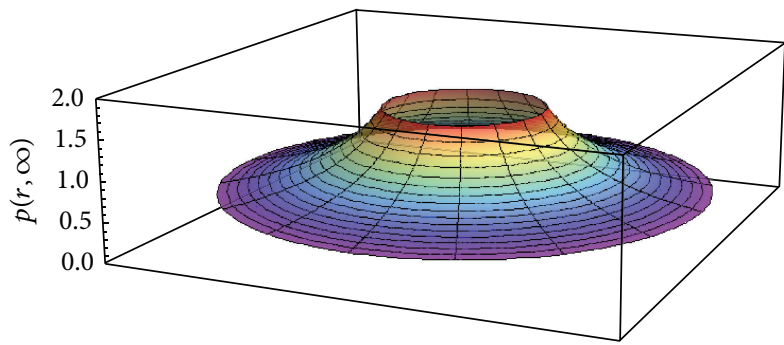

(c) $\gamma=0.1$

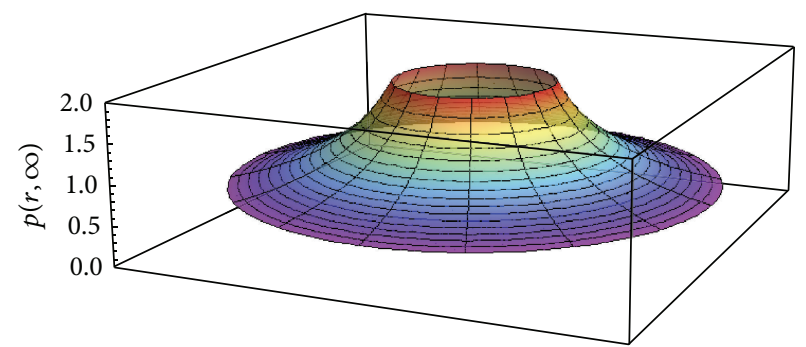

(b) $\gamma=0.01$

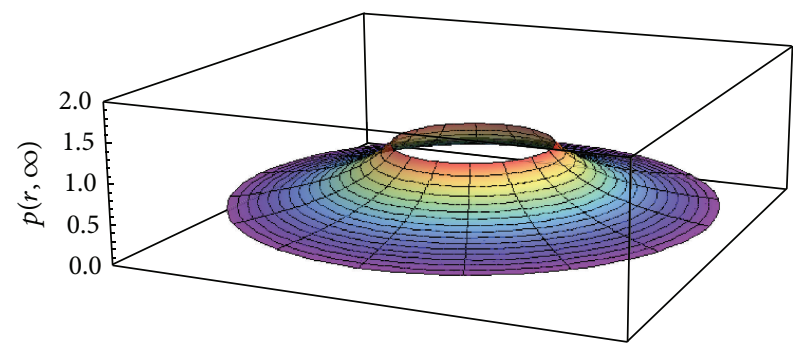

(d) $\gamma=1.0$

FIGURE 30: Contact pressure distributions as visualizations in polar coordinates. 


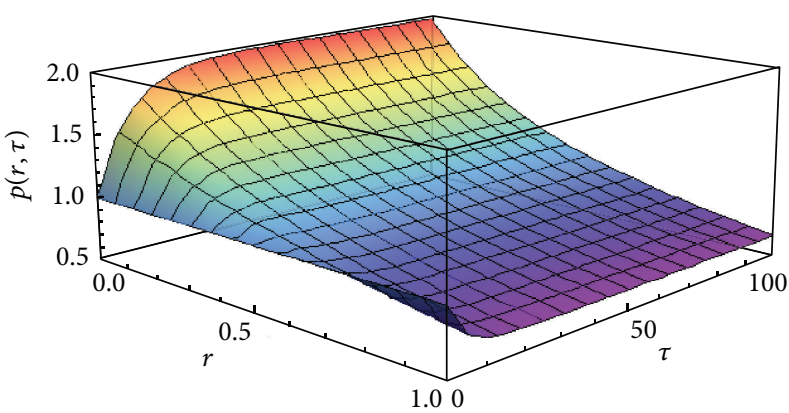

(a) $\gamma=0$

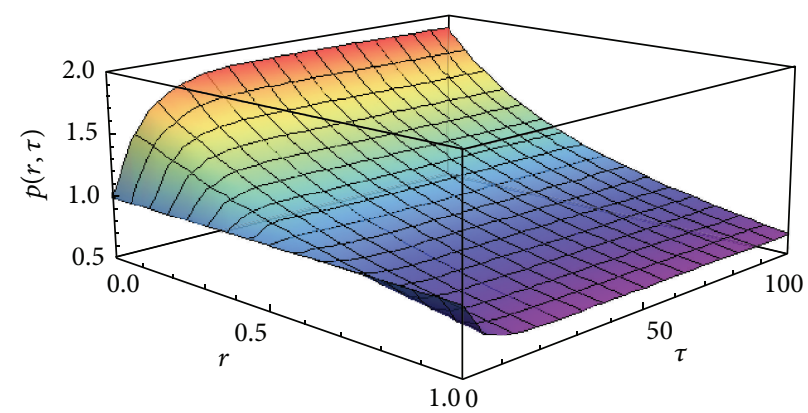

(b) $\gamma=0.01$

FIGURE 31: Changes of contact pressure distributions for various values of parameter $\gamma$.

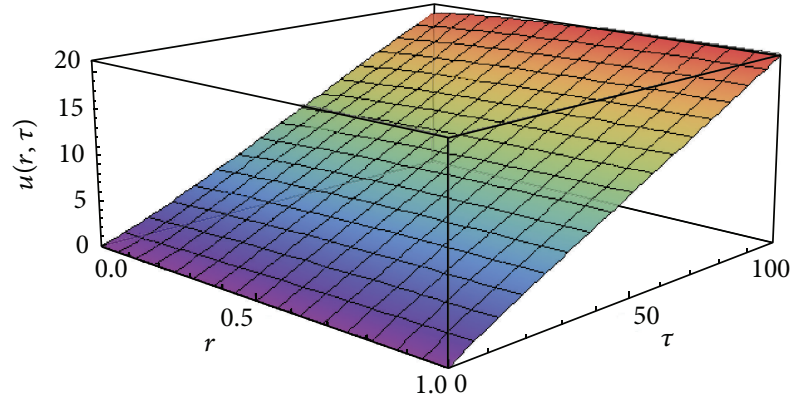

(a) $\gamma=0$

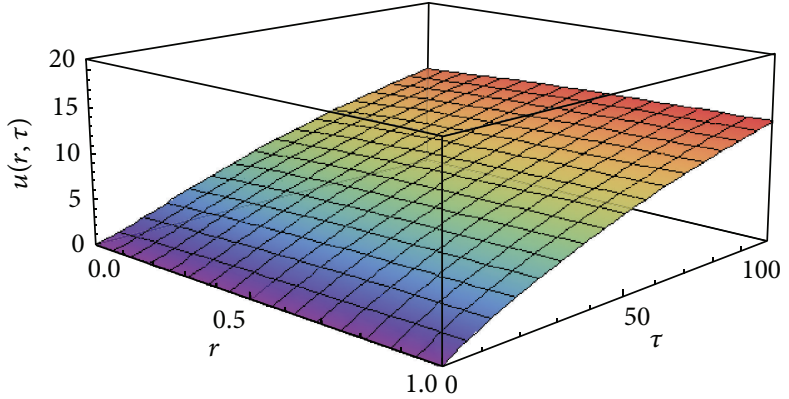

(b) $\gamma=0.01$

FiguRE 32: Changes of total wear distributions for various values of parameter $\gamma$.

with a relatively high accuracy with the analytically obtained results, we showed a few regularities; namely, the wear of clutch friction linings was approximately proportional to time (also to the work of friction force), the actual value of torque transmitted by the clutch decreased during the wear processes, changes of contact pressure distributions agreed with the proposed mathematical model (pressures increased at the inner border of the contact surface, while they decreased at the outer contact surface). As can be seen, the proposed models describe well enough the considered phenomena; therefore the proposed models have been used in numerical simulations in a wider range of parameters of the considered system, using our own computer programs written in $\mathrm{C}++$. In numerical calculations we focused on a symmetrical system consisting of two identical clutch friction linings and different values of the system parameters; however the presented models enable analysis of a general nonsymmetric system of clutch friction linings.

The obtained results are a basis for formulating final conclusions of our studies. The proposed mathematical description of the clutch takes into account elasticity of the friction lining materials. Thus, it is possible to consider more thoroughly the wear processes of clutch lining materials, calculating wear distributions on the entire contact surface. During numerical analysis in a wider range of parameter changes it was possible to determine nonuniform contact pressure and wear distributions at the contact surface of the friction linings. The obtained results help us better understand the mechanisms of wear processes of clutch friction linings and can be used, for instance, in the strength analysis of these systems. In addition, taking into account changes of contact pressure distributions we can estimate more accurately the friction torque transmitted by the clutch.

In our model we assumed simplification that there is no torsional vibration when the two linings are brought into contact. However, in practice relative slippage between them often is unavoidable. Although studies discussed in this paper were carried out in stationary conditions, the proposed mathematical models can also be used for the analysis in nonstationary conditions (for nonconstant force pressed clutch or nonconstant relative sliding velocity). Finally, it can be noted that we combine these issues in a complex tribological system, although the analytical, numerical, and experimental studies presented in the paper were carried out separately. For these reasons a better forecast of the behavior of real systems of this type is possible. Moreover, the results can be extended and used in other disciplines of science.

In comparison to our previous papers, in this paper mathematical models have been extended and generalized. Research methodology and interpretation of experimentally obtained data have been presented accurately. Moreover, a more exact theoretical analysis and new numerical simulations have been illustrated and discussed.

\section{Conflict of Interests}

The authors declare that there is no conflict of interests regarding the publication of this paper. 

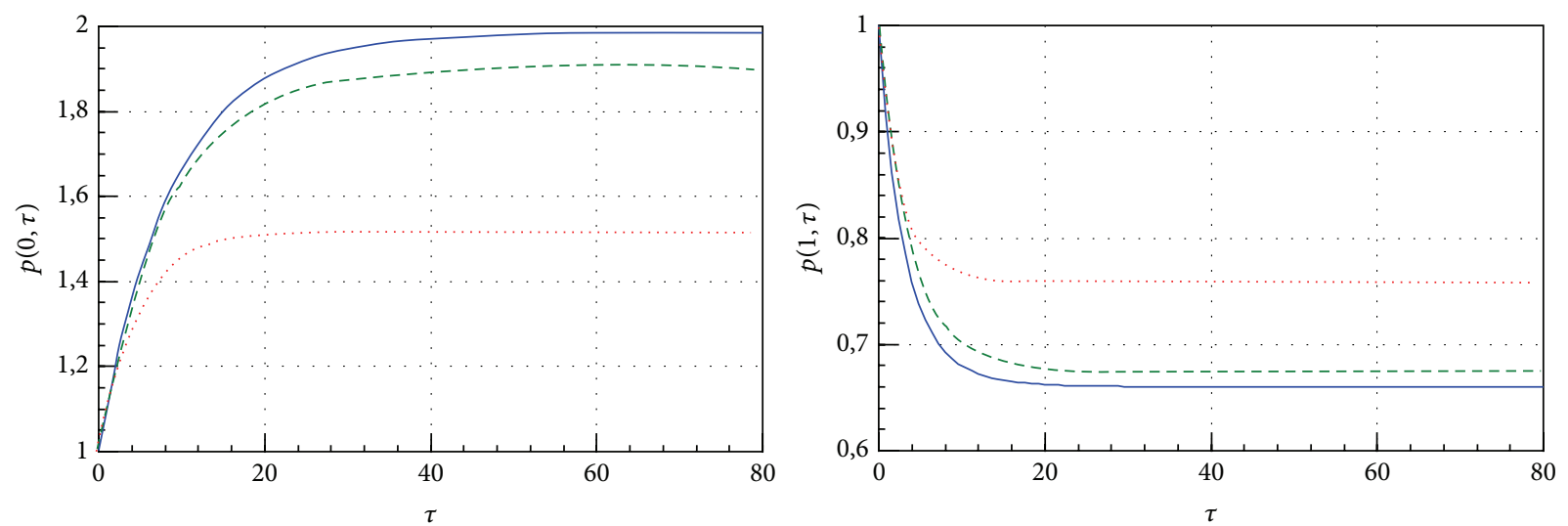

$$
\begin{aligned}
\gamma & =0 \\
--\gamma & =0.01 \\
\cdots \cdots & \gamma=0.1
\end{aligned}
$$

$$
\begin{aligned}
\gamma & =0 \\
--\gamma & =0.01 \\
\cdots \cdots \gamma & =0.1
\end{aligned}
$$

FiguRE 33: Contact pressures on the surface borders.
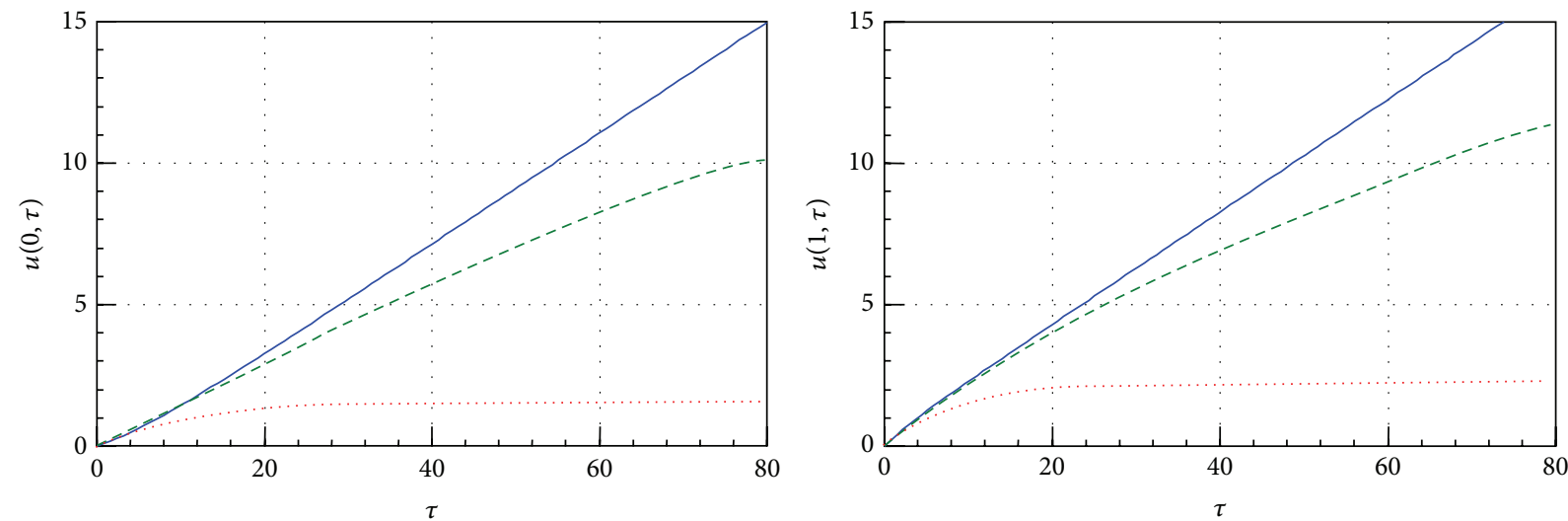

$$
\begin{aligned}
-\gamma & =0 \\
--\gamma & =0.01 \\
\cdots \gamma \gamma & =0.1
\end{aligned}
$$

$$
\begin{aligned}
\gamma & =0 \\
--\gamma & \gamma=0.01 \\
\cdots \cdots \quad \gamma & =0.1
\end{aligned}
$$

FIGURE 34: Wears on the contact surface borders.

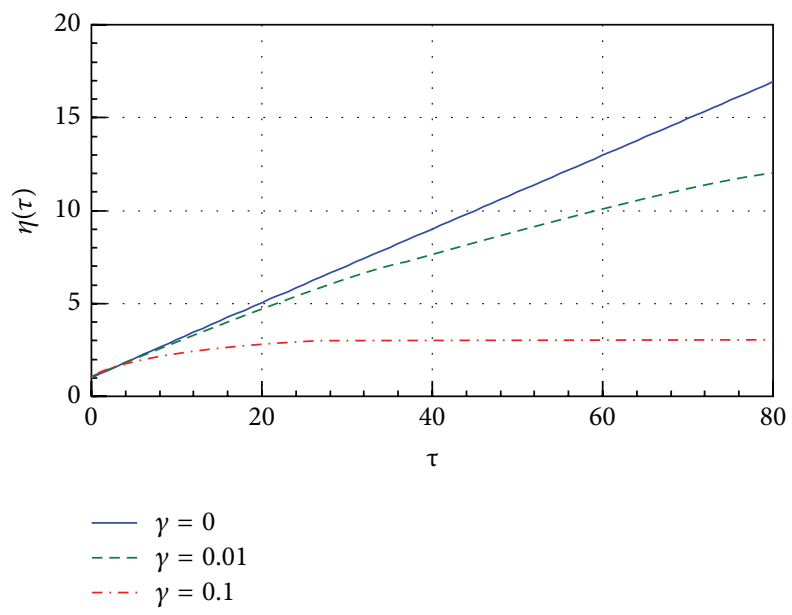

Figure 35: Decrease of the distance between clutch shields. 


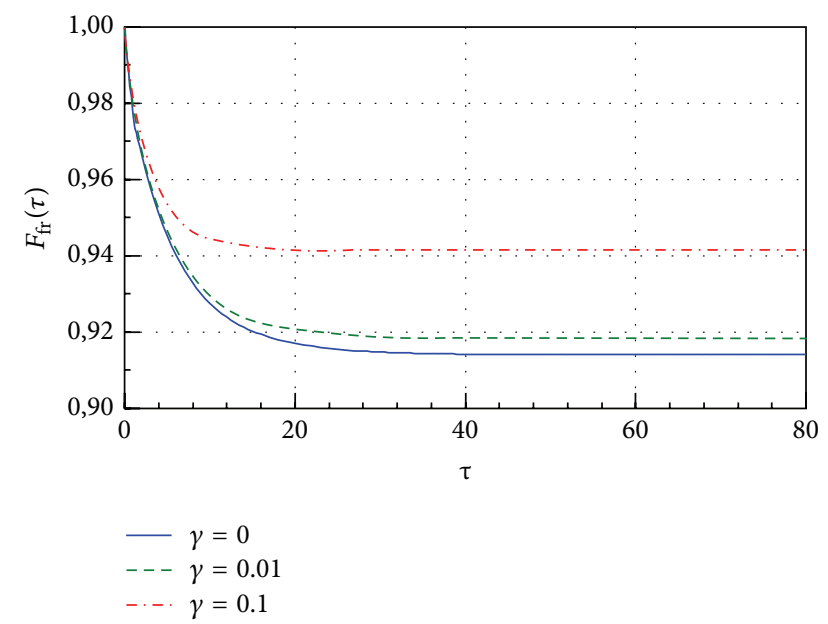

FIGURE 36: Changes of friction torque transmitted by the clutch.

\section{Acknowledgment}

The work has been supported by the National Science Foundation of Poland under Grant MAESTRO2 no. 2012/04/A/ ST8/00738 for years 2012-2016.

\section{References}

[1] D. Dowson, History of Tribology, Longman, London, UK, 1979.

[2] J. A. Williams, "Wear and wear particles-some fundamentals," Tribology International, vol. 38, no. 10, pp. 863-870, 2005.

[3] M. Azeem Ashraf, B. Sobhi-Najafabadi, Ö. Göl, and D. Sugumar, "Numerical simulation of sliding wear for a polymerpolymer sliding contact in an automotive application," International Journal of Advanced Manufacturing Technology, vol. 41, no. 11-12, pp. 1118-1129, 2009.

[4] P. J. Blau, "Fifty years of research on the wear of metals," Tribology International, vol. 30, no. 5, pp. 321-331, 1997.

[5] J. F. Archard, "Contact and rubbing of flat surfaces," Journal of Applied Physics, vol. 24, no. 8, pp. 981-988, 1953.

[6] N. H. Kim, D. Won, D. Burris et al., "Finite element analysis and experiments of metal/metal wear in oscillatory contacts," Wear, vol. 258, no. 11-12, pp. 1787-1793, 2005.

[7] N. Stalin-Muller and K. van Dang, "Numerical simulation of the sliding wear test in relation to material properties," Wear, vol. 203-204, pp. 180-186, 1997.

[8] M. Öqvist, "Numerical simulations of mild wear using updated geometry with different step size approaches," Wear, vol. 249, no. 1-2, pp. 6-11, 2001.

[9] P. Põdra and S. Andersson, "Simulating sliding wear with finite element method," Tribology International, vol. 32, no. 2, pp. 7181, 1999.

[10] S. K. Rhee, "Wear equation for polymers sliding against metal surfaces,” Wear, vol. 16, no. 6, pp. 431-445, 1970.

[11] S. K. Rhee, "Wear mechanisms for asbestos-reinforced automotive friction materials," Wear, vol. 29, no. 3, pp. 391-393, 1974.

[12] Z. Barecki and S. F. Scieszka, "Computer simulation of the lining wear process in friction brakes," Wear, vol. 127, no. 3, pp. 283305, 1988.
[13] D. Pavelescu and M. Musat, "Some relations for determining the wear of composite brake materials," Wear, vol. 27, no. 1, pp. 9197, 1974.

[14] S. M. Hsu, M. C. Shen, and A. W. Ruff, "Wear prediction for metals," Tribology International, vol. 30, no. 5, pp. 377-383, 1997.

[15] J. Awrejcewicz and Y. Pyryev, "Thermoelastic contact of a rotating shaft with a rigid bush in conditions of bush wear and stick-slip movements," International Journal of Engineering Science, vol. 40, no. 10, pp. 1113-1130, 2002.

[16] J. Awrejcewicz and Y. Pyryev, "Influence of tribological processes on a chaotic motion of a bush in cylinder-bush system," Meccanica, vol. 38, no. 6, pp. 749-761, 2003.

[17] M. V. Gavrikov and R. L. Mazing, "The hereditary-ageing model of wear and its application to problems with monotonically increasing contact zone," Treniye i Iznos, vol. 9, no. 2, pp. 274279, 1988 (Russian).

[18] O. V. Maksymuk, "Dependence of wear of anisotropic plates on their thickness," Materials Science, vol. 36, no. 4, pp. 620-624, 2000.

[19] O. V. Maksymuk, "Wear of thin coatings on solid bodies in the presence of an intermediate layer," Materials Science, vol. 37, no. 1, pp. 144-149, 2001.

[20] Y. Pyryev and A. Yevtushenko, "The influence of the brakes friction elements thickness on the contact temperature and wear," Heat and Mass Transfer, vol. 36, no. 4, pp. 319-323, 2000.

[21] P. Yu, Dynamics of contacting systems with heat production, friction phenomenon and wear processes [Post-doctoral thesis], Lodz University of Technology, Lodz, Poland, 2004, (Polish).

[22] A. Yevtushenko and P. Yu, "The applicability of a hereditary model of wear with an exponential kernel in the onedimensional contact problem taking frictional heat generation into account," Journal of Applied Mathematics and Mechanics, vol. 63, no. 5, pp. 795-801, 1999.

[23] I. V. Kragelsky, M. M. Dobychin, and V. M. Kombalov, Fundamentals of the Numerical Analysis of Friction and Wear, Mashinostroenie, Moscow, Russia, 1977, (Russian).

[24] N. Strömberg, L. Johansson, and A. Klarbring, "Derivation and analysis of a generalized standard model for contact, friction and wear," International Journal of Solids and Structures, vol. 33, no. 13, pp. 1817-1836, 1996.

[25] LA. Galin, Contact Problem of Elasticity and Viscoelasticity, Science, Moscow, Russia, 1980, (Russian).

[26] A. V. Blyumen, "The problem of the kinetics of friction and wear processes and methods for their analytical description," in Computational-Experimental Methods for Estimating Friction and Wear, pp. 277-33, Nauka, Moscow, Russia, 1980, (Russian).

[27] A. G. Kuzmenko, "Contact problems allowing for wear for cylindrical sliding supports," Treniye i Iznos, vol. 2, no. 3, pp. 502-512, 1981 (Russian).

[28] A. A. Yevtushenko and R. D. Kulchytsky-Zhyhailo, "Simplified solution for elliptical-contact problem with wear," International Journal of Engineering Science, vol. 35, no. 14, pp.1327-1334, 1997.

[29] I. Páczelt and Z. Mróz, “On optimal contact shapes generated by wear," International Journal for Numerical Methods in Engineering, vol. 63, no. 9, pp. 1250-1287, 2005.

[30] I. Páczelt and Z. Mróz, "Variational approach to the analysis of steady-state thermo-elastic wear regimes," International Journal for Numerical Methods in Engineering, vol. 81, no. 6, pp. 728-760, 2010.

[31] I. Páczelt and Z. Mróz, "Numerical analysis of steady thermoelastic wear regimes induced by translating and rotating 
punches," Computers \& Structures, vol. 89, no. 23-24, pp. 24952521, 2011.

[32] D. Hilding, A. Klarbring, and J. Petersson, "Optimization of structures in unilateral contact," ASME Applied Mechanics Reviews, vol. 52, no. 4, pp. 139-160, 1999.

[33] J. Awrejcewicz and D. Grzelczyk, "Applicability of a hereditary integral wear model with exponential kernel memory function in a friction connection of elastic shields of a friction clutch," International Journal of Applied Mechanics and Engineering, vol. 15, no. 3, pp. 609-617, 2010.

[34] J. Awrejcewicz and D. Grzelczyk, "Modeling and analyti$\mathrm{cal} /$ numerical analysis of wear processes in a mechanical friction clutch," International Journal of Bifurcation and Chaos, vol. 21, no. 10, pp. 2861-2869, 2011. 


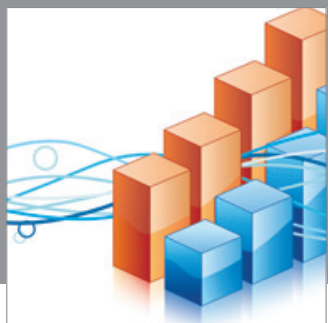

Advances in

Operations Research

mansans

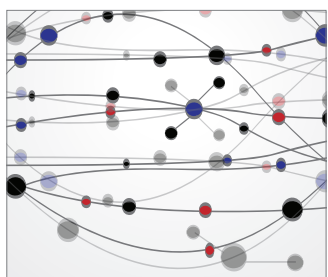

The Scientific World Journal
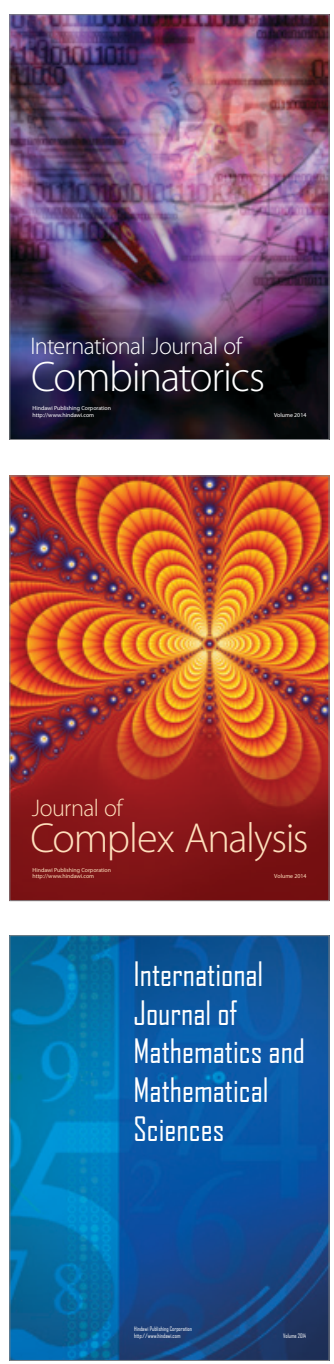
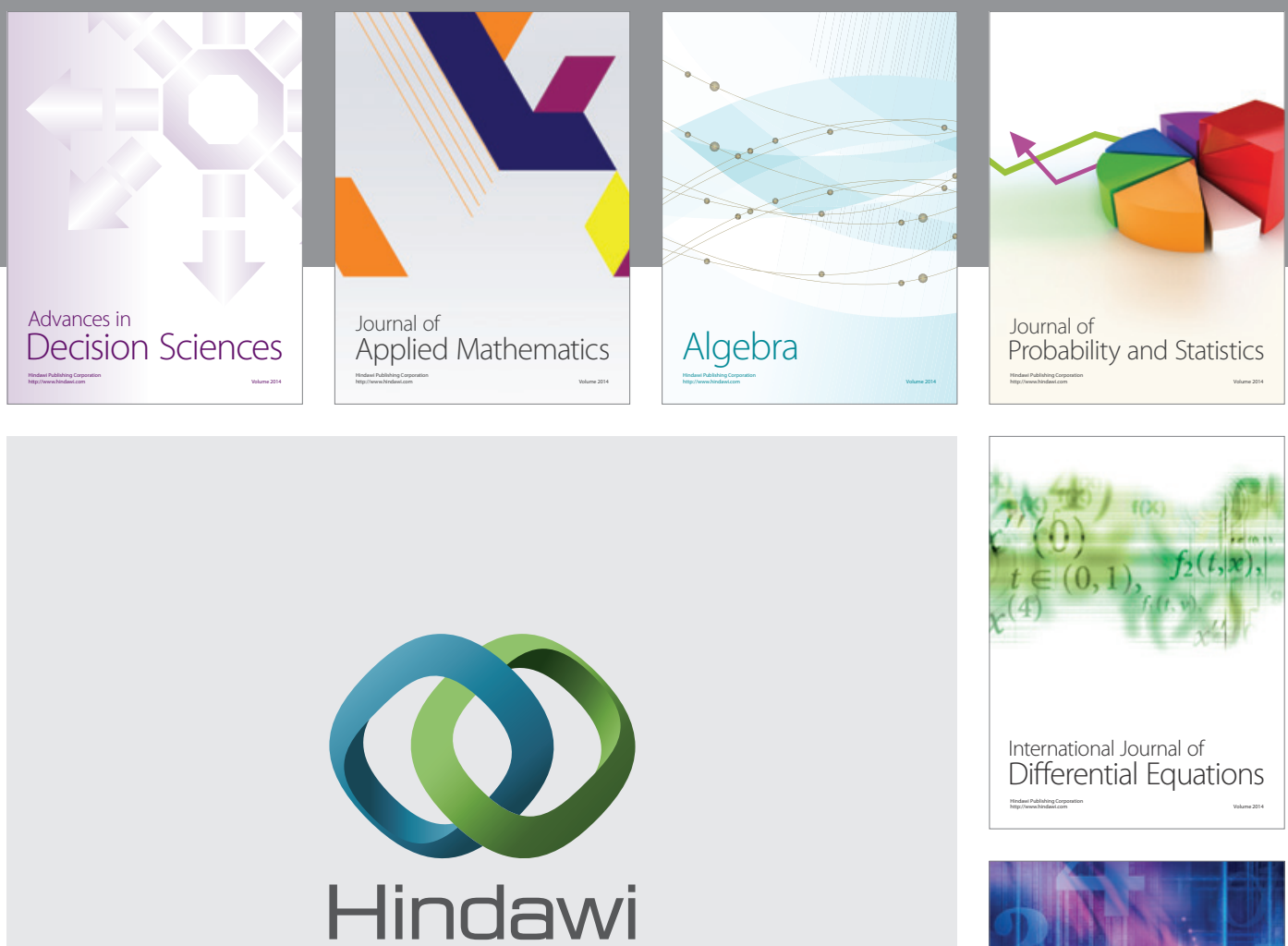

Submit your manuscripts at http://www.hindawi.com
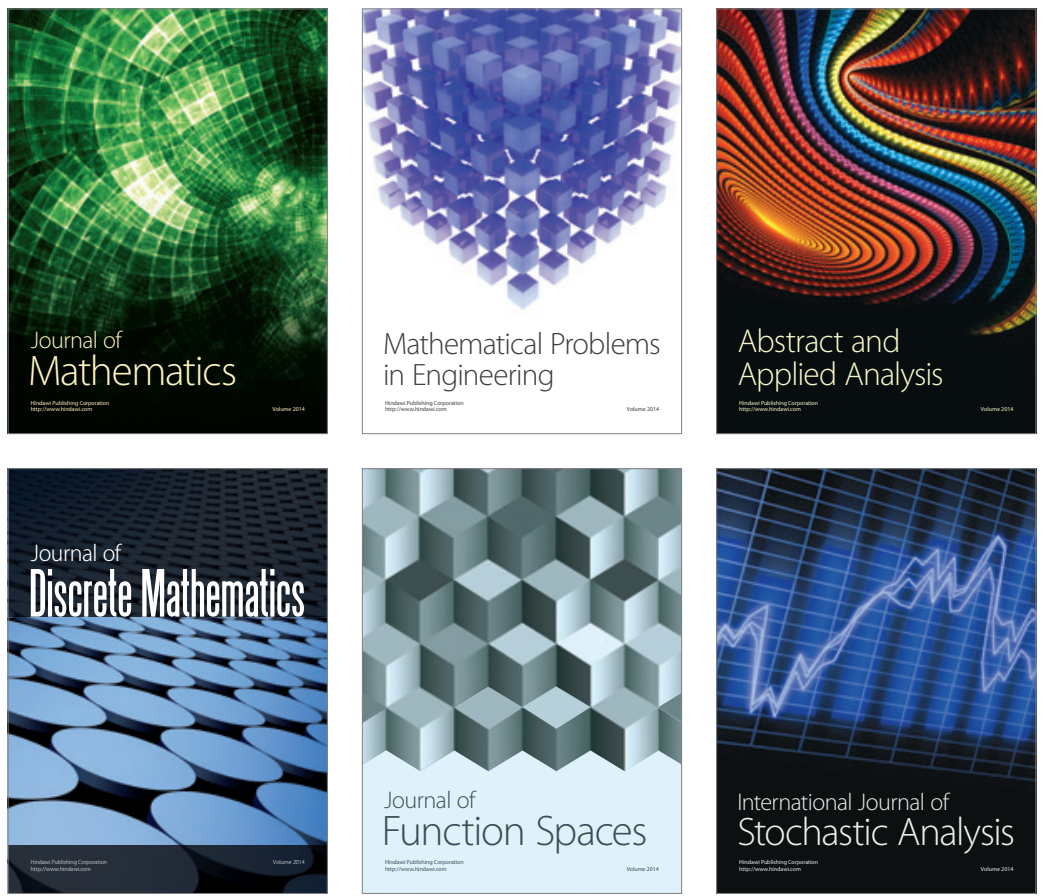

Journal of

Function Spaces

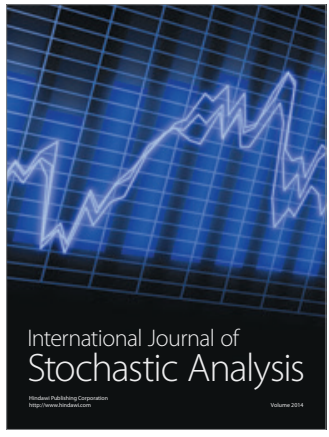

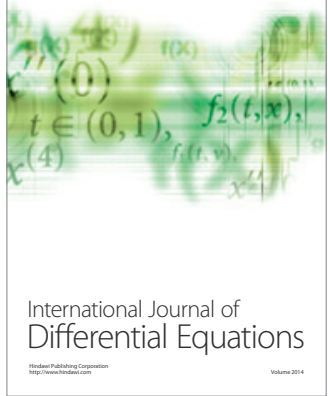
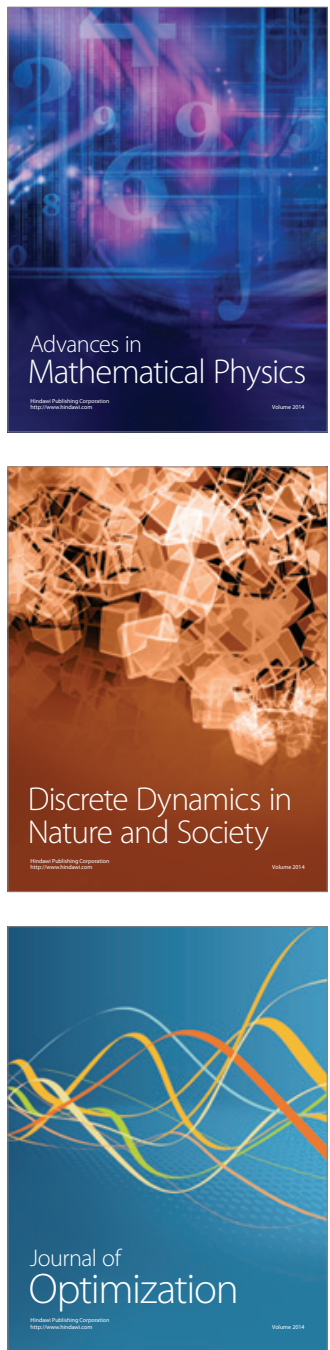